\title{
1 Brain-wide mapping of neural activity mediating collicular-dependent behaviors
}

Arnau Sans-Dublanc ${ }^{1,2 *}$, Anna Chrzanowska ${ }^{1,2 *}$, Katja Reinhard ${ }^{1,2,3}$, Dani Lemmon ${ }^{1,6}$, Gabriel Montaldo ${ }^{1,4}$, Alan Urban ${ }^{1,2,3,5}$ and Karl Farrow ${ }^{1,2,3,4 \#}$

1. Neuro-Electronics Research Flanders. Leuven, Belgium.

2. Department of Biology, KU Leuven. Leuven, Belgium.

3. VIB. Leuven, Belgium.

4. Imec, Leuven, Belgium.

5. Department of Neurosciences, KU Leuven. Leuven, Belgium.

6. Faculty of Pharmaceutical, Biomedical and Veterinary Sciences, University of Antwerp. Antwerp, Belgium.

* These authors contributed equally to this work

14 


\section{Abstract}

16 Neuronal cell-types are arranged in brain-wide circuits to guide behavior. In mice, the superior 17 colliculus is comprised of a set of cell-types that each innervate distinct downstream targets.

18 Here we reveal the brain-wide networks downstream of four collicular cell-types by combining 19 functional ultrasound imaging (fUSi) with optogenetics to monitor neural activity at a resolution 20 of $\sim 100 \mu \mathrm{m}$. Each neuronal group triggered different behaviors, and activated distinct, partially 21 overlapping sets of brain nuclei. This included regions not previously thought to mediate 22 defensive behaviors, e.g. the posterior paralaminar nuclei of the thalamus (PPnT), that we 23 show to play a role in suppressing habituation. Electrophysiological recordings support the 24 fUSi findings and show that neurons in the downstream nuclei preferentially respond to 25 innately threatening visual stimuli. This work provides insight into the functional organization 26 of the networks governing defensive behaviors and demonstrates an experimental approach 27 to explore the whole-brain neuronal activity downstream of targeted cell-types. 


\section{Introduction}

Different behavioral tasks rely on distinct networks of neurons distributed across the brain. Insights into how specific cell-types are linked to sensation and behavior have seen some great advances through the application of molecular technologies, providing a list of critical circuit elements ${ }^{1}$. On the other hand, computational understanding have been gained by comparing large-scale measurements of brain-wide activity with sensory inputs and behavior ${ }^{2-5}$. However, in mammals the link between individual cell-types, large scale neuronal activity and behavior remains unclear. In the superior colliculus (SC), there is evidence for a strong relationship between individual cell-types and behavior ${ }^{6-10}$. Here, we use this relationship to delineate the cell-type specific brain-wide functional networks that lie downstream of the SC.

In mice, the SC is a major hub of visual processing, where the superficial layers of the SC receive direct sensory inputs from $>85 \%$ of the retinal output neurons ${ }^{11}$. The retino-recipient neurons in the SC contain at least six sets of genetically identified cell-types with distinct anatomy and visual response properties ${ }^{12-16}$. Different cell-types project to different sets of targets, including nuclei of the thalamus and midbrain, thereby forming a putative structural basis for the relationship between cell-types and distinct behavioral properties $6,7,9,12,13,17,18$.

Optogenetic activation of cell-types in the retino-recipient layers of the colliculus has provided insight into the relationship between output circuits of the SC and behavior. For instance, activation of neurons that project to the pulvinar (LP) has been shown to induce arrest behavior in mice ${ }^{7,17}$, while activation of neurons projecting to the parabigeminal nucleus (PBG) leads to flight behavior ${ }^{7}$. These optogenetically induced behaviors resemble the reactions of mice to visual stimuli that mimic avian predators ${ }^{19,20}$. These experiments suggest that activation of neurons early in the visuo-motor circuits of the SC leads to downstream activity and behaviors that are comparable to the network activity and behaviors triggered by natural visual stimuli. But our view of these circuits remains limited to the specific circuits that have been investigated to date.

In rodents, combinations of cell-type specific stimulation and whole brain recordings using functional magnetic resonance imaging (fMRI) have provided insights into the relationship between cell-types and brain-wide network activity ${ }^{21,22}$. In relation to the SC, fMRI studies in humans have provided evidence that the pathway linking the $S C$ to the amygdala (AMG) via $\mathrm{LP}$ is involved in the processing of visual threats ${ }^{23-25}$. However, recording techniques such as fMRI suffer from limited resolution, which makes it difficult to clearly assign activity to small brain nuclei, in particular in small mammals ${ }^{21,26}$. Functional ultrasound imaging (fUSi) has been developed to study brain-wide activation patterns at a spatial and temporal resolution in awake mice that makes it practical to follow neural activity in most nuclei of the brain at a resolution of $\sim 100 \mu \mathrm{m}{ }^{27-32}$. In addition, its compact size allows for parallel interventions such as optogenetic activation or local neuronal recordings in awake behaving animals ${ }^{31,33}$.

By combining fUSi with optogenetics (opto-fUSi), we reveal in this study the neural networks through which information is routed after activation of different cell-types in the SC. We unravel the differences in the spatial and temporal organization of network activation depending on 
the cell-type and link these to differences in evoked behaviors. Opto-fUSi allows us to identify new brain areas that link sensory inputs to behavioral output, and we demonstrate that these brain areas are also activated by threat-like visual stimuli. Finally, chemogenetic manipulations unravel the potential function of one newly identified group of nuclei, the posterior paralaminar nuclei of the thalamus (PPnT), in visually triggered aversive behaviors.

\section{Results}

\section{Different collicular cell-types trigger different defensive behaviors}

To understand the contributions of different collicular cell classes to defensive behaviors, we optogenetically manipulated the activity of four genetically defined cell populations: 1) a population of excitatory neurons expressing CAMKII, referred to as CAMKII; 2) NTSR neurons that project to the LP, referred to as NTSR ${ }^{13}$; 3) the population of parvalbumin expressing neurons (PV) that consists of local interneurons and excitatory projections to LP, PBG and pontine gray (PG); 4) a set of inhibitory neurons (GAD2) that innervates the lateral geniculate nucleus (LGN) and PBG ${ }^{13}$. We restricted the expression of light-sensitive channelrhodopsin2 (ChR2) to the distinct cell classes in two ways. First, to express ChR2 in NTSR, PV and GAD2 neurons, we crossed Cre-expressing transgenic mouse lines (NTSRGN209-Cre, PV-Cre and GAD2-Cre) with a ChR2-reporter mouse line, Ai32 ${ }^{34-37}$. Second, CAMKII neurons were labeled with an adeno-associated virus (AAV) that carried ChR2 under the CAMKII promotor ${ }^{17}$ (see Methods). Control experiments were carried out with Crenegative litter mates. Histological analysis confirmed the layer-specific expression of ChR2 in different cell-types (Figure 1B), which was consistent with previous reports ${ }^{6-8,12,13,17}$.

To optically stimulate the colliculus, we stereotaxically placed an optical fiber over the medial portion of the superficial layers (Figure S1F). We performed the behavioral experiments in an open field setup ( $50 \mathrm{~cm} \times 50 \mathrm{~cm}$ box). At the beginning of each session mice were given a minimum of 2 minutes to freely explore the box before the experiments began (Figure 1A). Typically, animals were found to actively explore the box soon after being released into the arena, regularly moving from one corner to another (Movie S1; all the movies are listed an described in Table S1). Each trial was initiated when an animal entered the center of the arena, at which point we manually triggered the optogenetic stimulation. The stimulus consisted of blue light pulses ( $473 \mathrm{~nm}, 2 \mathrm{~ms}$ pulse width, $\sim 9.5-12.5 \mathrm{~mW} / \mathrm{mm}^{2}$ ) of either $1 \mathrm{~s}$ duration at 20 or $50 \mathrm{~Hz}$ or $4 \mathrm{~s}$ duration at $5 \mathrm{~Hz}$. CAMKII and NTSR neurons were stimulated at $20 \mathrm{~Hz}$, whereas GAD2 and PV neurons at $50 \mathrm{~Hz}$. We chose the stimulation frequency based on our preliminary behavioral observations (see Methods), and the documented firing rates recorded in response to natural visual stimuli ${ }^{6-8,13,17,38}$. We obtained similar results using either a 20 or $50 \mathrm{~Hz}$ (Figure 1) or $5 \mathrm{~Hz}$ (Figure S1A-F) stimulus. In the following paragraphs we focus on data obtained using the 20 or $50 \mathrm{~Hz} 1 \mathrm{~s}$ stimulus. 
A

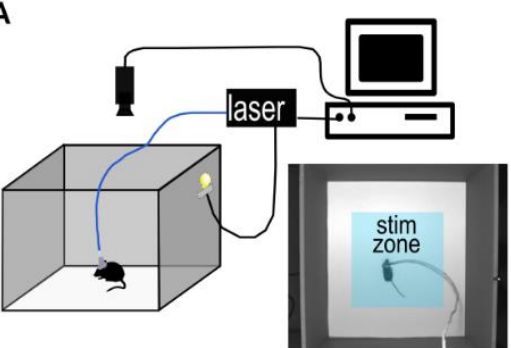

B

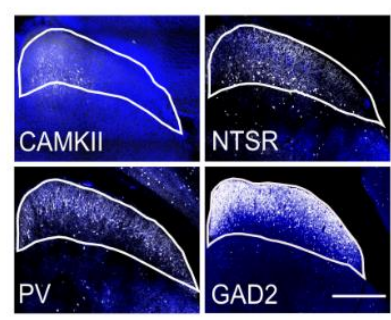

C

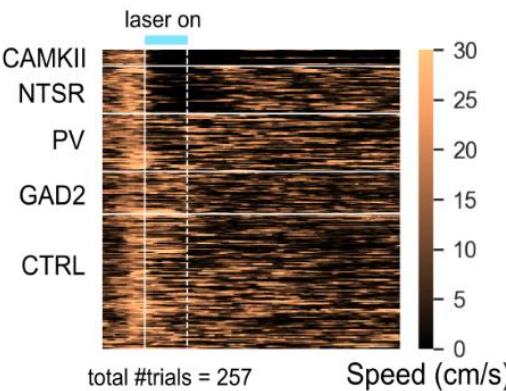

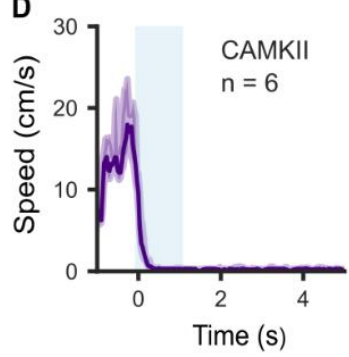
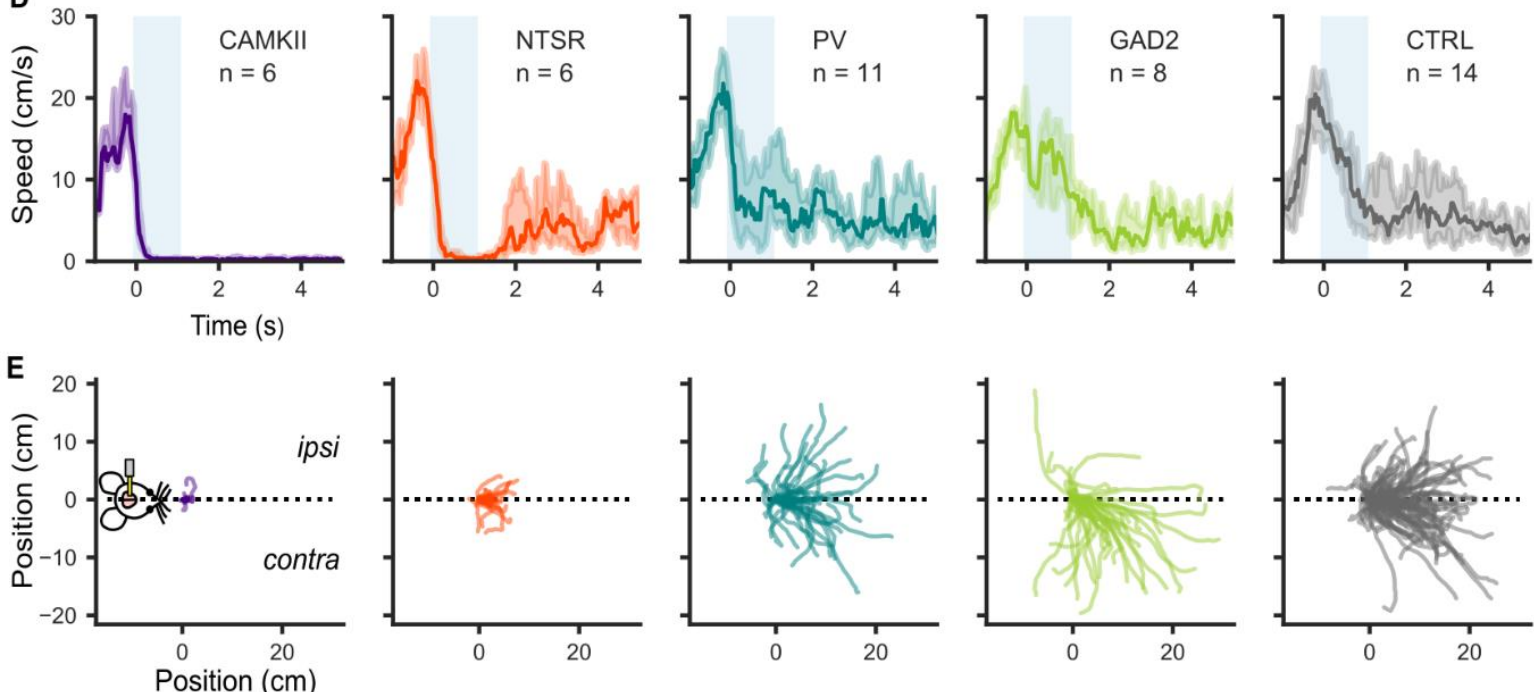

$\mathbf{F}$
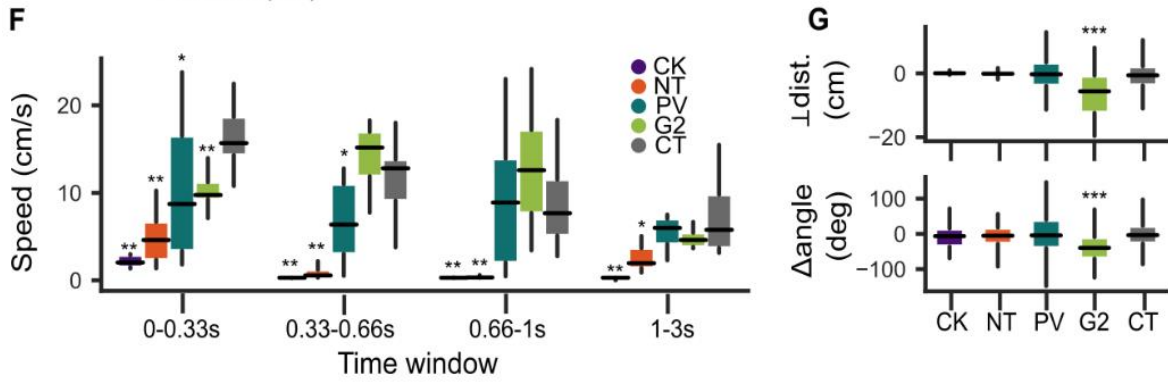

H

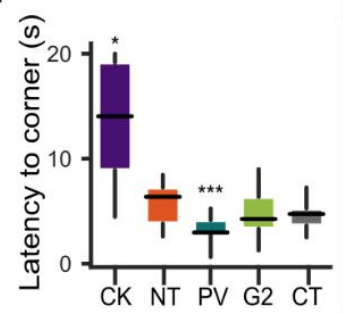

Figure 1. Different SC cell-types trigger different defensive behaviors. A. Schematic diagram of the open field setup for optogenetics and a video frame of mouse entering the stimulation zone in the center of the box (bottom right, stimulation zone is marked with blue rectangular shading). B. Coronal section showing expression of ChR2 in distinct cell lines. Scale bar, $500 \mu \mathrm{m}$. C. Heatmap of mice speeds during optogenetic stimulation trials. Values were obtained from the first experimental session for each animal. Horizontal white lines separate different mouse groups. Vertical solid and dashed white lines mark stimulus onset and offset, respectively. Light blue bar on the top marks the stimulus duration. D. Speed profiles. Each trace represents the median speed obtained from each mouse line. Shaded area represents the interquartile range. E. Mice trajectories during the stimulus duration (1s). Traces were aligned and rotated by the initial body position angle. CAMKII: $n=6,14$ trials, NTSR: $n=6,41$ trials, PV: $n=11$, 50 trials; GAD2: $n=8,43$ trials; CTRL $n=8,77$ trials. F. Speed quantification during chosen time windows. (0-0.33s: CAMKII $p=0.0003$, NTSR $p=0.0003$, PV $p=0.026$, GAD2 $p=0.004 ; 0.33-0.66 s:$ CAMKII $p=0.0003$, NTSR $p=0.0004$, PV $p=0.03$, GAD2 $p=0.10 ; 0.66-1 \mathrm{~s}$ : CAMKII $p=0.003$, NTSR $p=0.0004$, PV $p=0.34$, GAD2 $p=0.10 ; 1-3 \mathrm{~s}:$ CAMKII $p=0.0003, N T S R=0.007, P V p=0.38, G A D 2 p=0.18)$ G. Quantification of preferred body position at the stimulation offset, represented as a change of angle (bottom; CAMKII $p=0.26$; NTSR $p=0.31$; PV $p=0.45$; GAD2 $p=0.00002$ ) and perpendicular distance (top; CAMKII $p=0.27$; NTSR $p=0.29$; PV $p=0.38$; GAD2 $p=0.00002$ ), both in reference to $X$ axis (dashed line in E). H. Quantification of latency to a corner (CAMKII $p=0.003$; NTSR $p=0.15 ; P V p=0.013 ;$ GAD2 $p=0.49$ ). All data points are averaged over mice, except in $G$ where the data points are averaged over trials. Significance between control and each mouse line was tested using Mann-Whitney U-test (alpha $=0.05$ ). Box-andwhisker plots for F-H show median, interquartile range and range. ${ }^{*} p<0.05,{ }^{* *} p<0.01,{ }^{* * *} p<0.001$. 
Activation of each neural population led to distinct behavioral responses, that ranged from stopping (CAMKII and NTSR neurons) to directed movement (PV and GAD2 neurons; Figure 1). To capture differences in the triggered behavior, we first looked at the speed dynamics (Figure 1C-D and Figure 1F). All experimental groups responded with a drop in speed during the first $333 \mathrm{~ms}$ following the start of the stimulation (Figure 1F; Mann-Whitney U-test compared with control, CAMKII: $p=0.0003$; NTSR1: $p=0.0003 ; P V: p=0.026$; GAD2: $p=0.004$ ), whereas control mice did not show any identifiable change in behavior (Movie S2). An animal was determined to have stopped if its speed dropped below $1.5 \mathrm{~cm} / \mathrm{s}$ for at least $0.5 \mathrm{~s}$. Activation of CAMKII neurons resulted in particularly long stopping events that lasted for up to $19.8 \mathrm{~s}$ (Figure S1H, Movie S3; median stopping duration: $9.21 \mathrm{~s}, \mathrm{IQR}=[8.02,12.65]$ ), whereas stimulation of NTSR neurons resulted in mice stopping during the $1 \mathrm{~s}$ stimulus and resuming locomotion shortly after stimulus offset (median stopping duration $1.69 \mathrm{~s}, \mathrm{IQR}=[1.42$, 1.95]; Figure $\mathrm{S} 1 \mathrm{H}$, Movie $\mathrm{S} 4$ ). Activation of $\mathrm{PV}$ cells caused mice to slow down, but rarely led to a full stop (Figure S1I). Instead, their behavior was characterized by active movement towards one of the corners (Figure $1 \mathrm{H}$, Movie S5; median latency: $2.96 \mathrm{~s}, \mathrm{IQR}=[2.7,4.16]$; Mann-Whitney U-test compared with control, $p=0.01$ ). Animals with $\mathrm{ChR2}$ expression in GAD2 neurons showed a tendency to increase their speed during the stimulation (Figure 1F; MannWhitney U-test compared with control, GAD2: $\mathrm{p}=0.10$ ). Interestingly, this was accompanied by movement contralateral to the stimulated hemisphere (Figure $1 \mathrm{~F}-\mathrm{G}$ ) that manifested itself as turning (Movie S6, Figure 1G Bottom, median $\Delta$ angle: - $39^{\circ}$, IQR=[-70.01, -9.36]; Mann-Whitney U-test compared with control, GAD2: $\mathrm{p}=0.00002$ ), or a whole-body drift quantified as the perpendicular to the distance traveled in the first second relative to the axis of motion before the stimulus (Movie S7, Figure 1G Top; median perpendicular distance: $-5.66 \mathrm{~cm}$, IQR=[12.36,-0.72]; Mann-Whitney U-test compared with control, GAD2: $p=0.00002)$. Taken together, these findings suggest that each collicular cell-type makes a different contribution to behavior that can broadly be characterized as defensive or orienting.

\section{Brain-wide functional ultrasound during optogenetic stimulation in mice}

In order to assess the brain-wide neural activity that drives the different behaviors observed above, we developed a chronic preparation that allowed us to combine functional ultrasound brain imaging (fUSi) and optogenetics, in awake head fixed animals (Figure 2, A-C). fUSi reports neuronal activity indirectly by measuring changes in blood volume of the microvasculature ${ }^{32,39,40}$. To accommodate the optogenetic fiber and image as much of the brain as possible, a large cranial window that spanned a single hemisphere was implanted (AP +2 to $-6.5 ; L+1.25$ to -4.5 ). An optic fiber was pointed at the surface of the ipsilateral SC near the midline at an angle of $56^{\circ}$, approached from the contralateral side (Figure 2B; Methods). All animals included in the fUSi experiments were tested for behavior before each imaging session. 
A
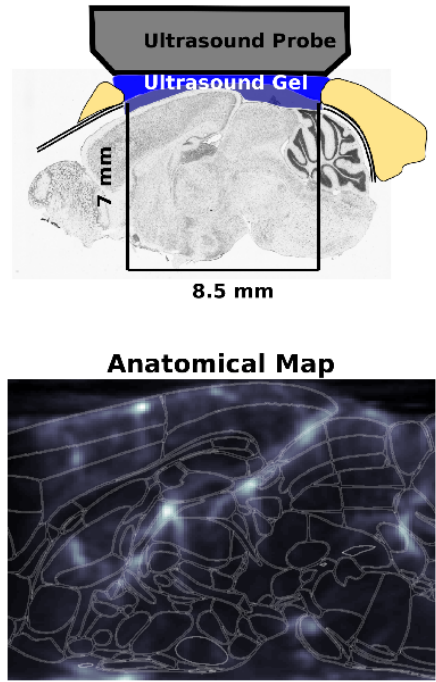

Fon
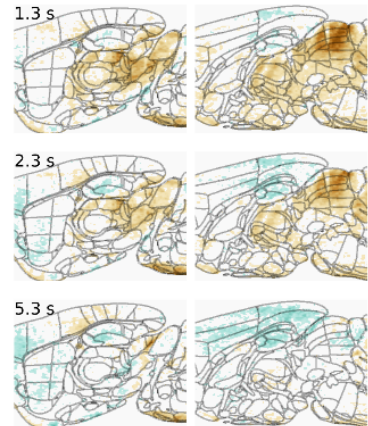

B

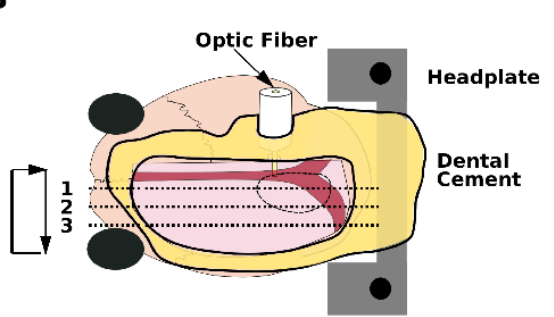

c

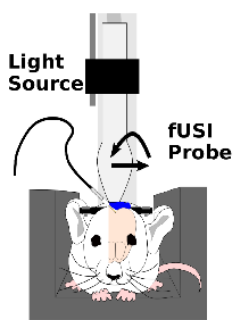

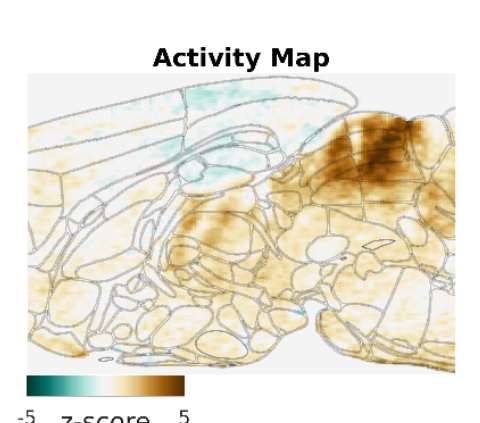

G

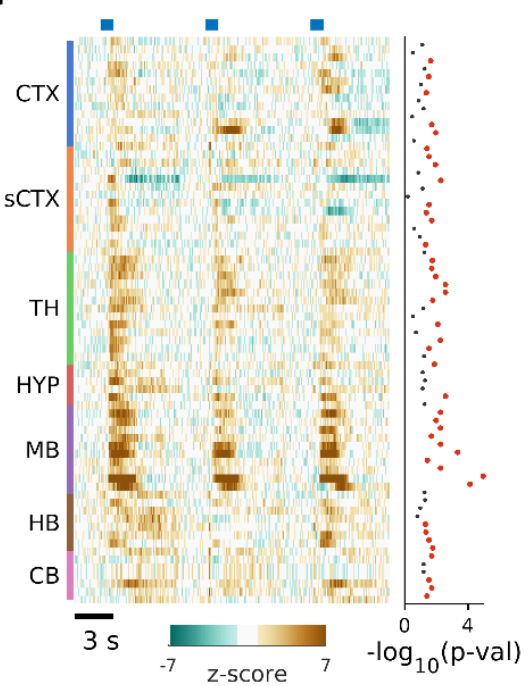

E

Regional Mean

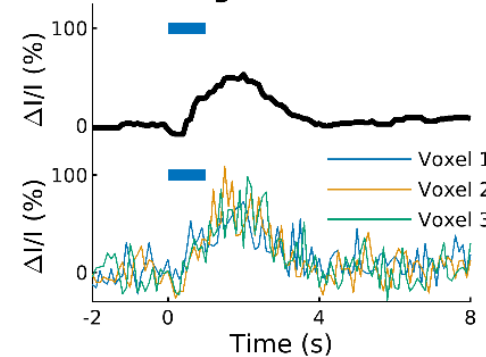

H

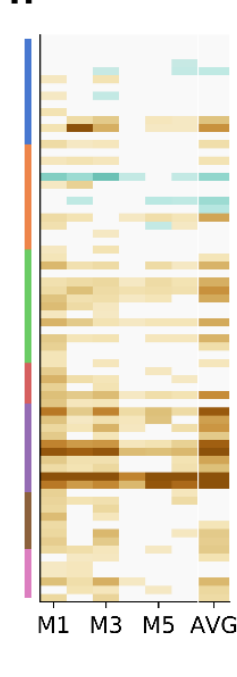

I

$4.25 \mathrm{~mm}$

$3 \mathrm{~mm}$

$1.75 \mathrm{~mm}$

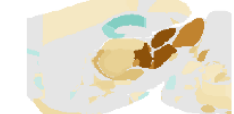

$0.5 \mathrm{~mm}$

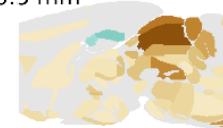

J

CAMKII

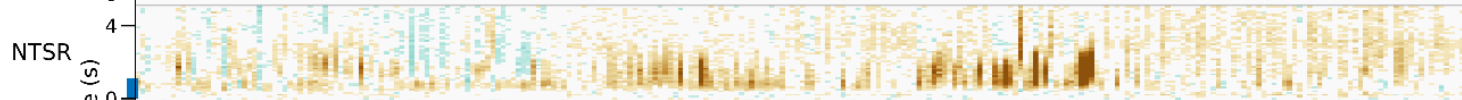
$\mathrm{PV}^{\mathrm{E}^{0}}{ }^{0}$ GAD2

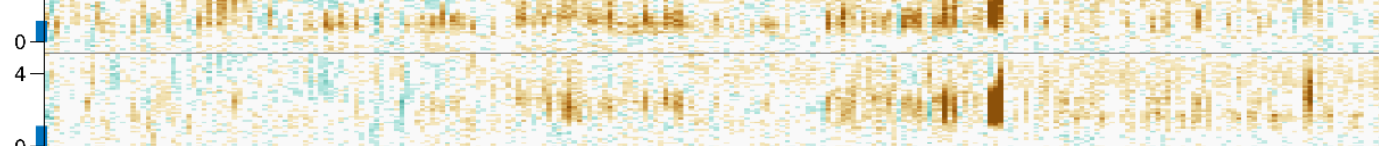

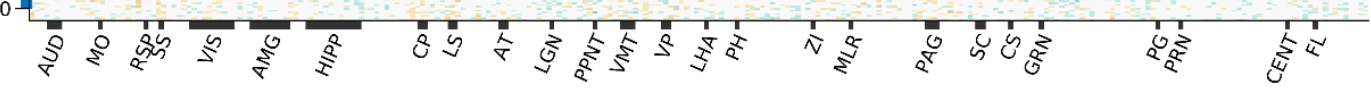


Figure 2. Functional ultrasound imaging of awake mice during optogenetic stimulation. A. Scheme of a sagittal cross-section of the chronic preparation. B. Top-view scheme of a chronic cranial window with implanted optic-fiber cannula inserted at $56^{\circ}$. C. Schematic of experimental set-up for awake imaging. D. Left. Example sagittal section of a blood volume map registered to the Allen Mouse brain reference atlas (thin gray lines). Right. Voxel to voxel normalized response to optogenetic stimulation of plane shown in left panel, registered to the Allen Mouse brain reference atlas (thin gray lines). E. Bottom: relative hemodynamic response curves to the optogenetic stimulation of three example voxels in the intermediate superior colliculus. Top: mean response of the intermediate superior colliculus. Blue lines indicate duration of optogenetic stimulation. F. Two example sagittal planes from the activity maps of a single animal. G. Left: Standardized responses of a selection of 72/264 segmented areas. Mean responses are shown for 3 different mice. Response for each mouse is an average of 6 trials. Blue lines indicate duration of optogenetic stimulation. black thick line indicates optogenetic stimulation. Right: Inactive (gray) and active (red) areas colored based on significance threshold corrected for multiple comparisons $(p<0.05)$. H. Mean response of each segmented area shown in G during the $2 \mathrm{~s}$ after the start of the stimulus for 6 different NTSR mice and the average across all mice. Areas considered not significant $(p>0.05)$ are set to zero in the average. I. Projection of the average activity vector from $\mathrm{H}$ onto a map of the mouse brain. J. Average time course of each of the 264 segmented areas for each stimulated cell population. Black bars along the bottom indicate span of the labeled brain regions.

CTX: cortex, sCTX: cortical subplate, TH: thalamus, HYP: hypothalamus, MB: midbrain, HB: hindbrain, CB: cerebellum

AUD: Auditory cortex, MO: Motor cortex, RSP: Retrosplenial cortex, SS: Somatosensory cortex, VIS: Visual cortex, AMG: Amygdala complex, HIPP: Hippocampus, CP: Caudatoputamen, LS: Lateral septum, AT: Anterior thalamus, LGN: Lateral geniculate nucleus, VMT: Ventromedial thalamus, VP: Ventral posterior thalamus, LHA: Lateral hypothalamic area, PH: Posterior hypothalamic area, ZI: Zona incerta, MLR: Mesencephalic locomotor region, CS: Superior central nucleus raphe, GRN: Gigantocellular reticular nucleus, PG: Pontine gray, PRN: Pontine reticular nuclei, CENT: Cerebellar lobuli, FL: Flocculus

144 Neural activity was monitored with a fUSi probe positioned over the craniotomy parallel to the

145 long axis of the animal. Sagittal planes were imaged sequentially, each spanning the entire 146 depth of the brain, where the probe was stepped (250 um) along the medial-lateral axis (Figure

147 2A-C). While imaging each plane, the colliculus was optically stimulated. In each experiment, 148 each plane was imaged for two 20 s periods, when either a $1 \mathrm{~s}(20 \mathrm{~Hz}$ or $50 \mathrm{~Hz})$, or $4 \mathrm{~s}(5 \mathrm{~Hz})$

149 light stimulation was delivered via the implanted optic fiber $10 \mathrm{~s}$ after the imaging started. The 150 parameters of the light stimulation were the same as those used during the behavioral 151 experiments. Each mouse was imaged in 3-5 sessions that were separated by 48-72 152 hours. Each voxel was assigned to an individual brain region by performing a 3D rigid 153 registration of the series of sagittal images, obtained in the absence of visual stimulation (125 $154 \mu \mathrm{m}$ steps), to the Allen Mouse Brain Common Coordinate Framework version 3 (CCF v3) ${ }^{41}$ 155 (Figure 2D). We used a modified version that is comprised of 264 brain areas in one 156 hemisphere of the brain (Table S2).

157 To build a spatial map of brain activity, we compared, voxel by voxel, the hemodynamic signals 158 (DI/l, referred to as "activity") obtained during and after the optogenetic stimulus to a $10 \mathrm{~s}$ 159 period before the light stimulus (Figure 2D-F; Movie S8). The hemodynamic activity of all 160 voxels within each area were averaged to estimate the response for that region (Figure 1E and 161 G). Temporal traces were obtained for each mouse and compared (t-test corrected for false 162 discovery rate) to identify the areas that displayed a response (Figure 1G, H-I). Average 163 responses for each segmented area in each mouse line are shown in Figure 1J. The same 164 analysis was applied to low-frequency stimulation data (Figure S3). 
A
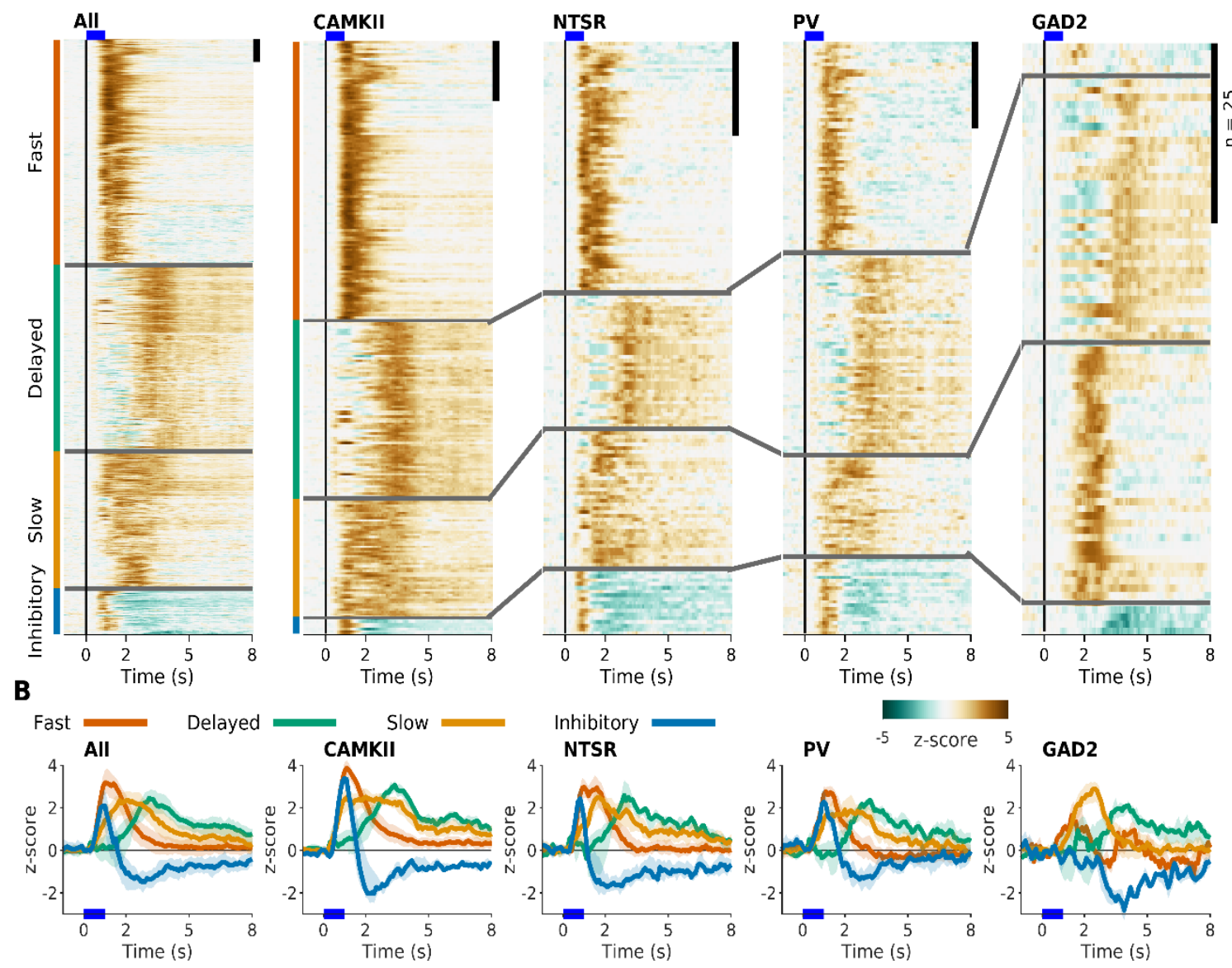

C
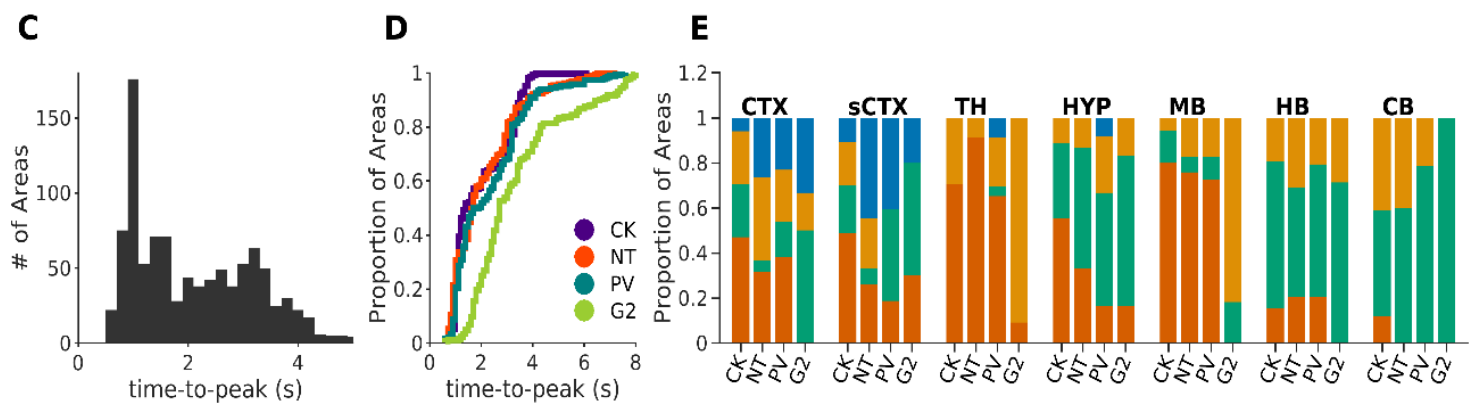

Figure 3 Distribution of temporal responses dynamics. A. Normalized responses to optic stimulation. Responses are organized into their respective clusters: Fast (orange), Delayed (green), Slow (yellow) and Inhibitory (blue). Black scale bar at top right of each panel represents 25 areas. Blue line represents the $1 \mathrm{~s}$ optical stimulus. Left Panel. Responses of all areas that had a statistically significant response across all cell populations $(n=659)$. Other panels are the active areas in each mouse line $(C K=246, N T=157, P V=170, G 2=82)$. B. Average response of each of clustered responses. C. Histogram of the time-to-peak of each active area in all mouse lines. D. Cumulative histogram of time-to-peak in each mouse line. E. Proportion of each response type sorted by brain area and mouse line.

\section{Distribution of temporal response properties}

166 We began our analysis of how distinct cell-types of the SC distribute information across the brain by looking at the temporal structure of the hemodynamic changes induced by the optical stimulation (Figure 3 ). We found that our $1 \mathrm{~s}$ optical stimulus caused a reliable set of temporal responses that could be grouped into 4 broad categories (see Methods). These four response types could be broadly described as: Fast, Delayed, Slow and Inhibitory (Figure 3A and B). The Fast responses were characterized by a relatively fast rise time $(1.27+/-0.42 \mathrm{~s})$, resulting in a transient response. The Delayed responses showed a clear delay with time to peak of 3.3 
$+/-0.79 \mathrm{~s}$. The Slow responding areas started their responses early but took longer to reach their peak $(2.1 \mathrm{~s}+/-0.70 \mathrm{~s})$ and showed a more sustained response $(1.78+/-1.30 \mathrm{~s})$. Finally, a set of responses that we will refer to as Inhibitory, showed a negative response. Inhibitory responses were commonly preceded by a very transient early positive response (time-topeak $=0.91+/-0.65 \mathrm{~s}$ : decay time $=0.52+/-0.18 \mathrm{~s}$ ) in each cell-class except the GAD2 (Figure $3 \mathrm{~A}$ and $\mathrm{BB}$ ).

The distribution of the different response types varied among the mouse lines. We found that the Fast responses were more common in CAMKII and NTSR mice (CK 47\%; NT 42\%; PV $35 \%$; G2 5\%). PV mice had a similar proportion of fast (35\%) and delayed (34\%) responses, while GAD2 mice had predominantly delayed (45\%) and slow (44\%) responses. In addition, inhibitory response types were more common in NTSR and PV mice, as compared to CAMKII and GAD2 (CK 3\%; NT 11\%; PV 13\%; G2 5\%). The almost complete absence of Fast responses in the GAD2 mice is evident in the distribution of the response latencies, estimated as the timeto-peak (Figure 3C-D). The distribution of latencies formed two broad groups, those responding within the first $2 \mathrm{~s}$, and those responding after $2 \mathrm{~s}$ (Figure $3 \mathrm{C}$ ). While the distribution of latencies is similar for CAMKII, NTSR and PV cell populations, and spanned the entire range of times, activation of inhibitory GAD2 neurons did not cause any early responses (Figure 3A and 3D).

Stimulating the $\mathrm{SC}$ neurons at a lower frequency $(5 \mathrm{~Hz}$ for $4 \mathrm{~s})$, generated similar temporal dynamics triggered by each neuronal population (fast, delayed, slow, inhibitory), but exacerbated the differences between them (Figure S3). Additionally, the change in frequency corresponded with a reversal in the sign of the response in some areas such as the visual cortex. The visual cortex had a fast positive response during high-frequency stimulus, but an inhibitory response during the low-frequency for each cell population (Figure 3 and Figure S3).

The distribution of the different response types across the major brain structures was relatively consistent between the different cell populations, except for slow responses (Figure 3E). Fast responses occurred mainly in the midbrain and thalamus, delayed activations took place in hypothalamus, hindbrain and cerebellum, and inhibitory responses in the cortex and cortical subplate. Slow responses were more homogeneously distributed. For example, CAMKII and NTSR had the largest proportion of slow responding areas in the cerebellum (23 and $24 \%$, respectively), while GAD2 had none in that structure.

\section{Different brain-wide activity patterns are triggered by each class of cells}

To understand how the activation of each neuronal population triggers distinct brain-wide networks, we compared the distribution of brain areas that had increased or decreased hemodynamic responses for each cell-type. Based on the temporal dynamics of the responses observed (Figure $3 C$ ), we performed this comparison in two distinct time windows, an early (0-2 s) and a late (3-8 s) phase (Figure 4A). Complete lists of the responsive areas to high- and low-frequency stimuli are provided in the supplementary materials (Table S3-S4). 3D movies of the activated brain areas can be found in the supplementary material for the early (Movie S9-12) and late phase (Movie S13-16). 
A
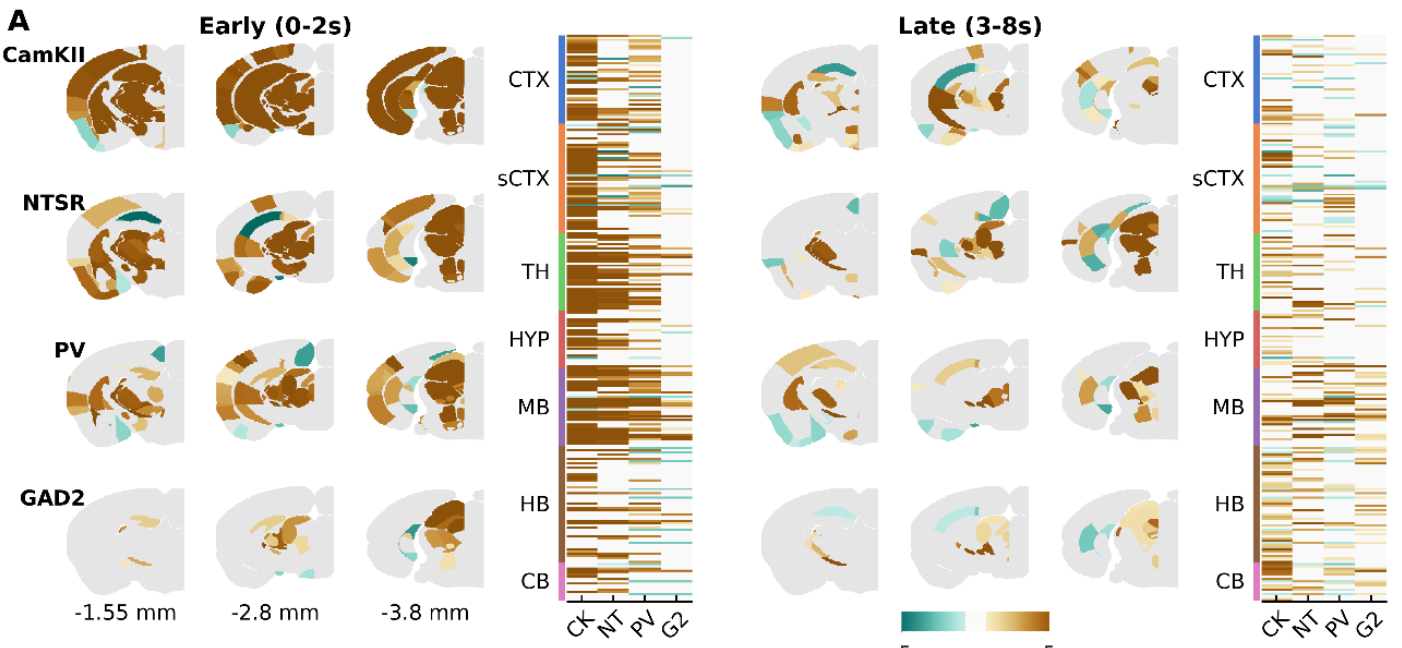

$\mathbf{B}$
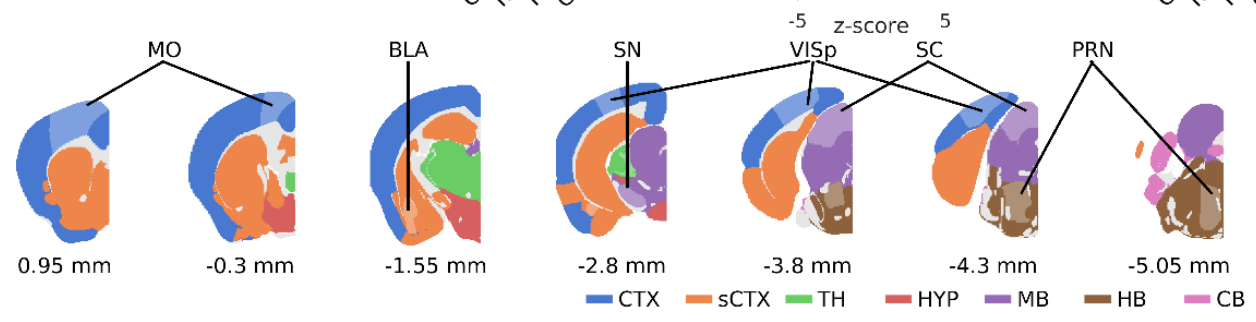

C

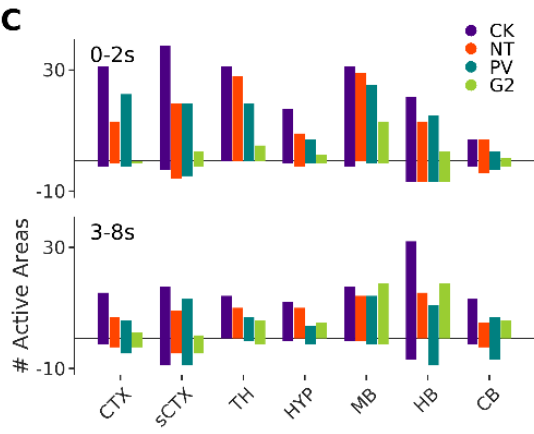

D

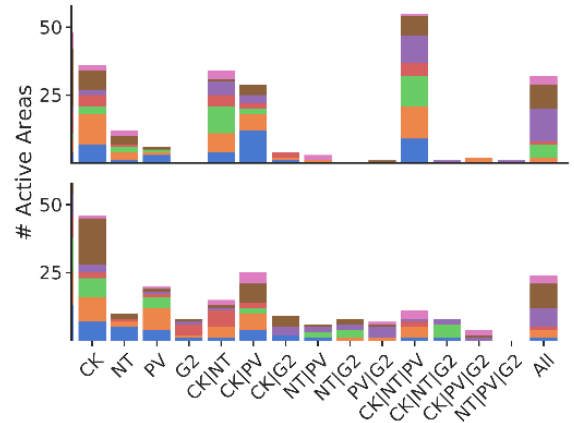

$\mathbf{E}$
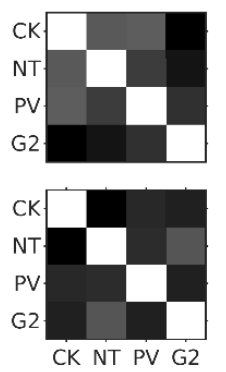

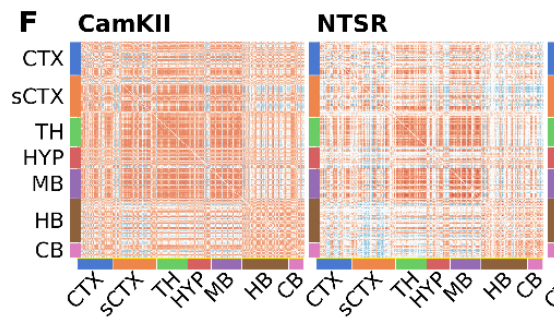

pv

GAD2

G

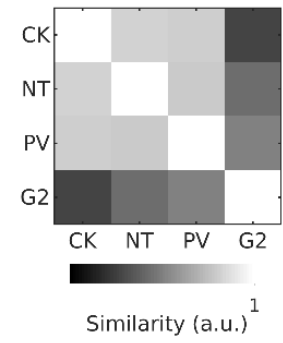

Figure 4. Cell-type specific activation of downstream pathways of the superior colliculus. A. Activation maps during early (Left, 0-2 sec) and late (Right, 3-8 sec) time windows. Three example coronal slices are shown for each mouse line. Active areas are shown in each plane with the mean $z$-score across mice (CK=6; NT=6; $P V=13 ; G 2=6$ ). Next to the activation maps, is the peak response of all areas active in at least one mouse line. B. Summary of the extent of the imaging locations and corresponding names. Imaging was done in 264 brain regions. Here we delineate the major brain areas and highlight a few single regions for orientation. A complete list of the segmented brain regions and abbreviations are presented in Table S2. C. Distribution of active areas during early and late phases. D. Quantification of shared areas across mouse lines during early (Top) and late (Bottom) phases. Areas included in one group are excluded from the others. E. Similarity matrix (between cell populations) of maximum activity during early (Top) and late (Bottom) phases. F. Pairwise Pearson correlation coefficients between the mean response traces of the 264 segmented areas during the $8 \mathrm{~s}$ after stimulus onset. $\mathbf{G}$. Similarity between the correlated hemodynamic responses in $\mathrm{F}$. 
214 The distribution of responsive areas across the brain in the early and late phases followed

215 different patterns for each of the cell classes (Figure 4A and C). Broadly, stimulating CAMKII,

216 NTSR and PV cell-types at high-frequency, resulted in more responses during the early phase

217 (CK=193; NT=138; $P V=129)$, as compared to the late phase (CK=142; NT=82; $P V=97)$. In

218 contrast, stimulating GAD2 cells resulted in 41 areas responding during the first two seconds,

219 followed by an additional 68 areas responding during the late phase. When we compared the

220 distributions of the active areas (Figure 4C), we found that stimulation of CAMKII evoked

221 responses in large portions of the CTX (80\%, 33/41 areas), sCTX (80\%, 41/51 areas), TH (80\%,

$22231 / 36$ areas) and MB (89\%, 33/37 areas) in the early phase, but was dominated by HB (72\%,

$22339 / 54$ areas) and CB (79\%, 15/19 areas) in the late phase. Stimulating the NTSR population

224 activated large portions of the MB (78\%), and $\mathrm{TH}(78 \%)$, during the early phase (other

225 structures ranged from 34\%-58\%) and had less but more distributed activity during the late

226 phase (CTX: 24\%, sCTX: 27\%, TH: 28\%, HY: 38\%, MB: 41\%, HB: 28\%, CB: 42\%). PV neurons

227 preferentially modulated the MB (MB: 70\%; others: $31-59 \%$ ) in the early phase, and the HB

228 (HB: 74\%; others: 22-43\%) in the late phase. In GAD2 mice, most areas were activated in the

229 MB in the early phase (MB: 38\%; others: 2-19\%). During the late phase, GAD2 activated more

230 areas across the whole brain, particularly in the MB (early/late; 38\% / 54\%), the HB (early/late;

231 19\% / 33\%) and CB (early/late; 16\% / 32\%). Low-frequency stimulation did not change the

232 overall distribution of responsive areas in early and late phases of the different cell-types

233 (Figure S4A). However, compared to high-frequency, PV and GAD2 mice had a noticeable

234 decrease of activated areas in both the early (high-frequency/low-frequency; PV: 129/72;

235 GAD2: 41/14) and late (high-frequency/low-frequency; PV: 97/73; GAD2: 68/9) phases.

236 Differently, NTSR mice had more responsive areas in both early and late phases, most noticeable in the HB during the late phase (high-frequency/low-frequency; HB: $28 \%$ / 93\%). Of note, the early phase of NTSR mice had a large increase of negatively modulated areas of the cortex by low-frequency stimulation, which had positive responses upon high-frequency stimulation (Figure S4A-B).

241 To gain insight into the different downstream networks, we next looked at the overlap between

242 the areas modulated by the different cell classes (Figure 4D-E). We found that in the early

243 phase up to 93 areas had shared activity between at least three of the neuronal populations,

24471 where shared by only two, and 54 areas were unique. Consistent with the fact that the

245 CAMKII population likely includes the other two excitatory cell-types, when two areas were

246 shared, in most cases it was between CAMKII and either PV (29) or NTSR (34) mice. During

247 the late phase, the specificity increased and only 47 areas where shared by three or more cell-

248 types, compared to the ones shared by two (70) or uniquely modulated (84). To measure how

249 similar the activated networks were from each other, we calculated the similarity between cell-

250 lines of the maximum activity during early and late phases (Figure 4E). We found that during

251 the early phase, the greatest similarity was between PV and NTSR with CAMKII (Figure 4E).

252 GAD2 showed the least similarity with the other cell lines. During the late phase, GAD2 and

253 NTSR mice showed the highest similarity towards each other (Figure 4E Bottom), and all other 254 pairings showed very low similarity. Low-frequency stimulation was characterized by a large 
increase in the number of areas solely activated by NTSR neurons (Figure S4E). Similarity analysis showed that, in the early phase, CAMKII and PV networks were the most similar (Figure S4D Top). In the late phase, the similarity pattern was conserved, with GAD2 and NTSR networks being the most similar (Figure S4D Bottom).

Finally, in order to compare the activated networks from a holistic point of view, we generated functional connectivity maps of the relationship between areas across the whole brain (Figure $4 \mathrm{~F})$. To do this, we first quantified the pairwise correlation across all active areas of each neuronal population. Then, we compared the resulting matrices to each other (Figure $4 G$ ). Broadly, correlations across the brain upon high-frequency stimulation followed similar patterns in CAMKII, NTSR and PV, and were clearly different from GAD2. More concretely, CAMKII, NTSR and PV mice all had a marked high level of correlation between areas of the $M B$ and TH. In GAD2 the highest correlations where between the MB, the HB and the CB. Under low-frequency stimulation, CAMKII and NTSR mice continued to have similar correlation patterns across the brain (Figure S4E-F), but there was a pronounced shift of the brain-wide correlations that showed increased correlations of the MB with the HY, $\mathrm{HB}$ and $\mathrm{CB}$ and a decrease with CTX and SCTX. The change in frequency did not affect the brain-wide correlations of PV mice, while the correlations for GAD2 became sparser and more localized within the different structures, making it the most differentiated cell-type (Figure S4F). Taken together, these results indicate that each collicular cell-type modulates a distinct brain-wide network.

\section{Defensive and fear related networks are differentially modulated by each cell class.}

To understand how the activation of each neuronal population triggers distinct aversive behaviors, we compared the activity patterns of each cell-type within a list of 30 areas that have been previously shown to mediate or modulate defensive behaviors (Figure $5 \mathrm{~A}$ ). This comparison showed that each neuronal population activated a different subset of areas or modulated the same areas in a different manner. For example, the central amygdala (CEA), the posterior medial and paraventricular hypothalamic areas $(\mathrm{PMH}, \mathrm{PVH})$, and the ventral tegmental area (VTA) were shared uniquely by cell classes that elicited freezing-like behaviors (CK, NT). On the contrary, the cuneiform (CUN), or the superior central nucleus (CS) were activated by all mouse lines but exhibited different temporal dynamics. The CUN had fast transient responses in CAMKII and NTSR and slower sustained responses in PV and GAD2 mice, whereas the CS had sustained responses in CAMKII and GAD2 but transient in NTSR and PV. Interestingly, there were also cases where different cell-types activated the same areas but in opposite directions. For example, areas of the ventral midline thalamus (RE and $X i)$, cingulate cortex (ACAd), and subthalamic nucleus (STN), had positive responses to CAMKII and NTSR types, but were dominated by negative responses in PV. Finally, a few areas were similarly activated by all mouse lines, namely the motor layers of the colliculus (SCi), the dorsal periaqueductal gray (PAGd) and the zona incerta (ZI), all with similar fast positive responses. When we compared the correlated activity across this group of areas (Figure 5B), and the similarity of the traces (Figure $5 \mathrm{C}$ ) across the different mouse lines, it confirmed that CAMKII and NTSR evoked the most similar responses compared to PV, and GAD2. Principal 
A

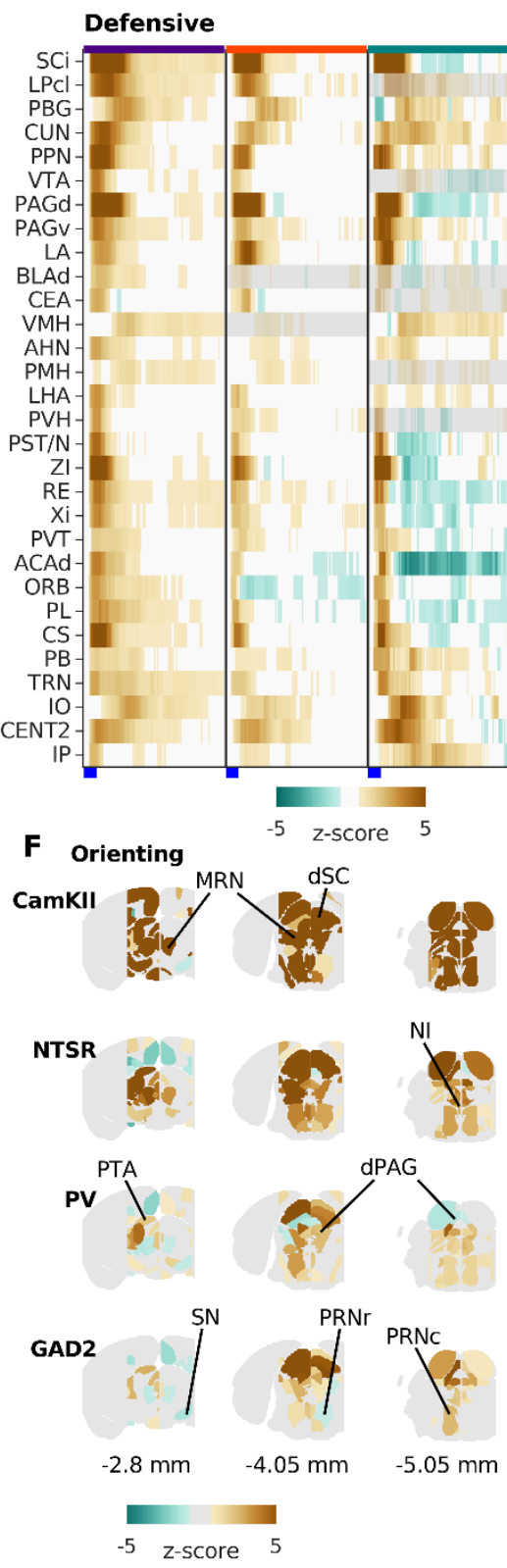

B

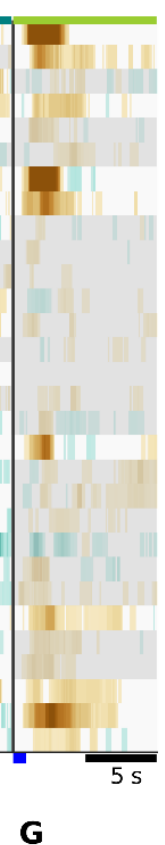

$\mathbf{G}$

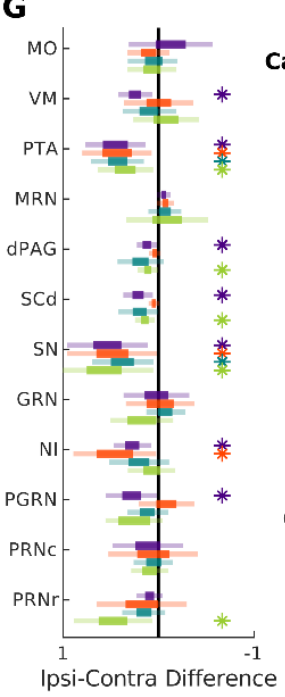

D

Ipsi-Contra Difference
C
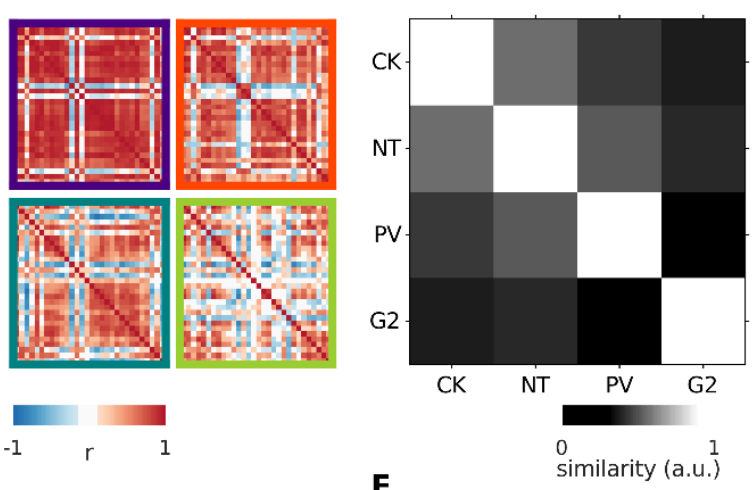

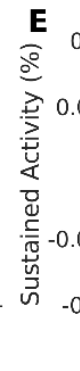
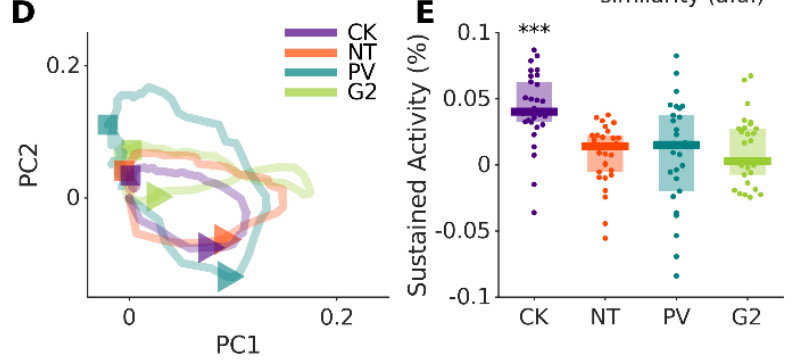

H

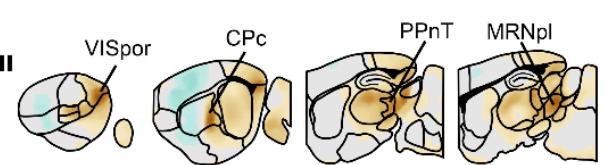

NTSR

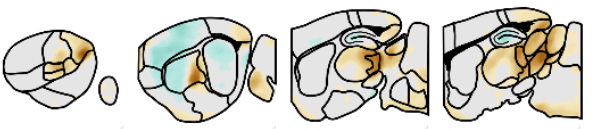

PV

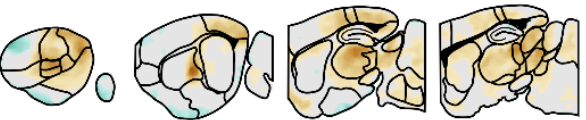

GAD2

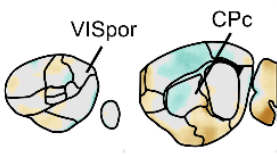

$4.25 \mathrm{~mm}$

$3.25 \mathrm{~mm}$

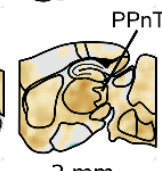

PPnT MRNpl

$2 \mathrm{~mm}$

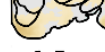

-3 z-score 3

Figure 5. Activity in behaviorally defined networks. A. Heatmap of the average responses of 30 nuclei commonly associated with defensive behaviors triggered the different cell populations. B. Correlation matrix of the responses in each cell populations. C. Similarity of the response properties of these 30 areas across cell populations. D. 2D Trajectories of the neuronal activity in these 30 brain areas. Triangle and square are the points on the trajectories $1.5 \mathrm{~s}$ and $5 \mathrm{~s}$ after the stimulus, respectively. E. The average sustained activity for each of the 30 areas after highfrequency stimulus of each cell population. Each dot represents a brain area. F. Comparison of the activity in the nuclei that lie within $2 \mathrm{~mm}$ of the midline, both ipsi- and contralateral to the optogenetic fiber stimulus. Each ipsicontra pair is shown when the difference is the highest within a $4 \mathrm{~s}$ window after stimulus onset $\mathbf{G}$. The percent difference in the fUSi signal between the ipsi- and contralateral brain areas. ${ }^{*}$ represent differences that were statistically significant ( $p<0.05$ permutation test, after correction for multiple comparisons). Light color lines represent the estimated 95\% confidence intervals. Dark portions represent the interquartile range. $\mathbf{H}$. Average pixelpixel maps of four sagittal sections from each neuronal population that highlight brain areas not commonly reported to mediate visually guided defensive behaviors. 
component analysis of the trajectories followed by the responses (Figure 5D), showed that CAMKII and NTSR evoked almost identical responses during the first 2 seconds after the stimulus onset, but then diverged into different paths. This was likely due to the more sustained activity evoked by the excitation of CAMKII neurons (Figure 5E). These results are consistent with the different collicular cell-types activating distinct behaviors through parallel functional networks.

\section{4}

305

306

307

308

309

310

311

312

313

314

315

\section{Asymmetric activity originating from the medial part of the superior colliculus}

Orienting behaviors, including eye, head and body movements can be controlled via contralateral projections of the SC to the medial pontomedullary reticular formation (MPRF) ${ }^{42,43}$. In mice the MPRF is comprised of a set of nuclei that includes the pontine gray and pontine reticular nuclei (PRN) that we were able monitor on both sides of the brain as they lie close to the midline. On the contrary, defensive behaviors are thought to be mediated mainly by ipsilateral pathways originating in the medial part of the SC ${ }^{44}$. Consistent with our stimulations targeting the medial part of the colliculus, we found that all cell-lines evoked asymmetric activations that where preferentially ipsilateral (Figure 5F-G). Also, activating GAD2 neurons generated the greatest number of asymmetries, including pontine areas such as the rostral part of the PRN, which is in line with that cell line being the only one triggering a change in orientation.

\section{Novel areas involved in collicular-driven aversive behaviors}

Visual inspection of brain-wide activity maps (Figure $5 \mathrm{H}$ ), revealed a few highly responsive nodes in areas that have not been previously studied in the context of collicular-driven defensive behaviors. The four areas that were most salient were the the caudoputamen, especially its caudal part (CPc); the postrhinal visual area (VISpor); the posterior lateral part of the midbrain reticular formation (MRNpl) and a group of thalamic areas surrounding the medial geniculate complex referred to here as the posterior paralaminar nuclei of the thalamus (PPnT) 45. The VISpor and CPC are known di-synaptic targets of the colliculus, via the pulvinar, but have not been implicated in guiding defensive behaviors ${ }^{46-49}$. The MRNpl and PPnT have not been previously described to receive mono- or di-synaptic inputs from retino-recipient neurons of the SC ${ }^{14}$.

\section{Correspondence between fUSi and neuronal activity}

To test to what degree fUSi signals correlate with the underlying spiking activity, we used Neuropixels probes to record from different parts of the brain and compare them to the fUSi responses observed for the same stimulus. We focused on NTSR cell population in Ntsr-Cre $x$ Chr2 mice. Animals were head-fixed on a treadmill or floating ball and neural activity was recorded while either optogenetically activating NTSR neurons with repeated trials of $1 \mathrm{~s} 20 \mathrm{~Hz}$ stimulation, or while viewing visual stimuli on a screen (Figure $6 \mathrm{~A}$ ). The recording probes were coated with a fluorescent dye (Dil) to visualize the recording locations post-hoc (Figure 6B). On some electrodes, we found spiking activity that was triggered by each of the 20 light pulses (Figure 6C Top). On other electrodes, while the response to the first light pulse was often strong, the responses to the subsequent pulses were weak or absent (Figure 6C middle and Bottom). A raster plot of all 384 recording electrodes for one trial of 20 light pulses are shown 


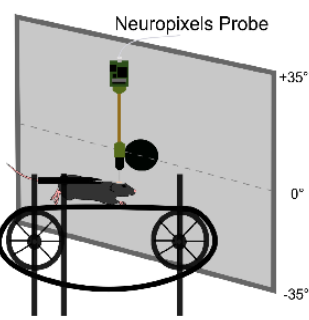

B

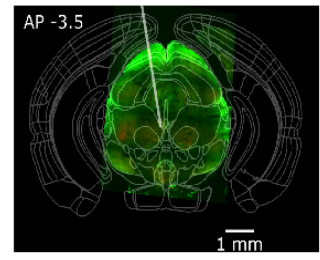

C

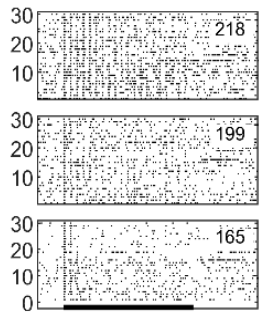

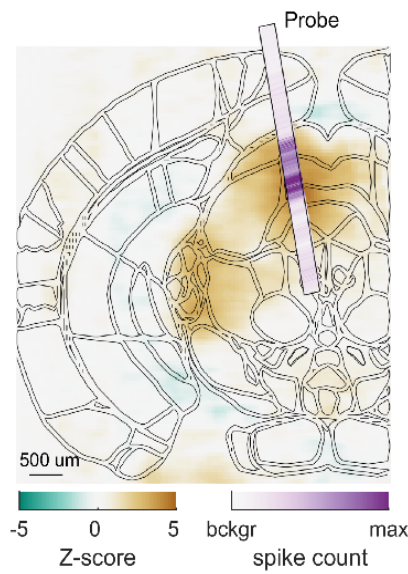

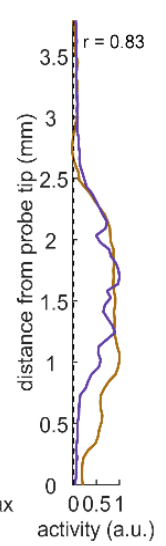

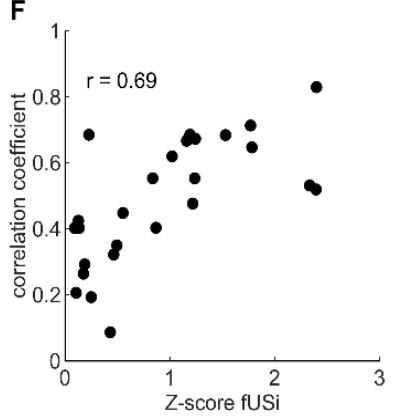

D
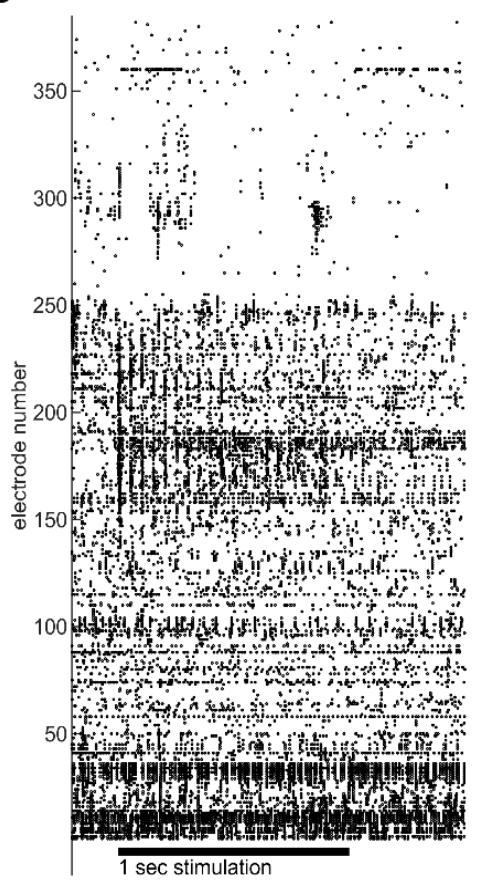

J

H

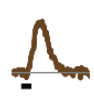

$\operatorname{sSC}{ }_{0}^{1} \mid$

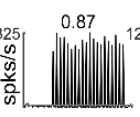

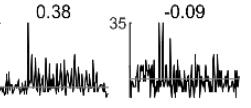
$-0.19$
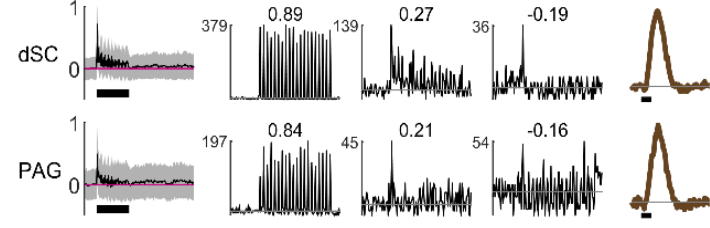

0.21
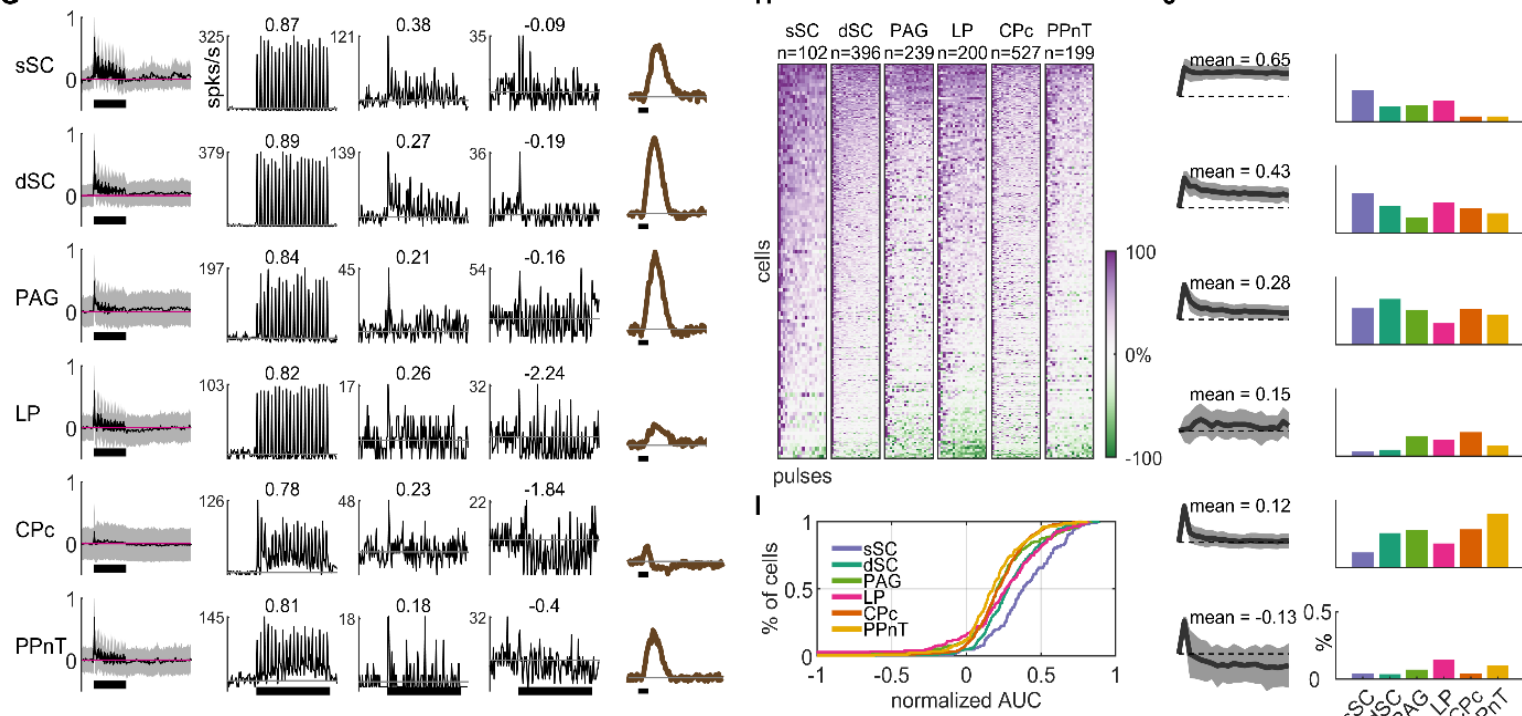

$=0.43$

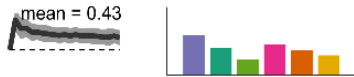

100

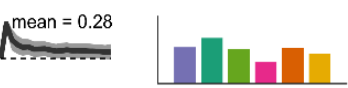

$0 \%$

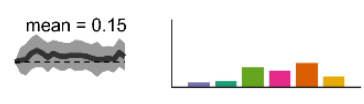

Figure 6. Correspondence of fUSi and spiking activity. A. Setup for Neuropixels recordings in awake, headfixed mice. B. Example histological section with Ntsr-Chr2 positive neurons (green) and the probe location indicated with a gray line. C. Raster of spikes for 30 repetitions of the optogenetic stimulus for three example electrodes. Stimulus time (1 s, 20 pulses) is indicated with a black bar. D. Raw spiking data on all 384 electrodes of the probe shown in B during a $20 \mathrm{~Hz}$ optogenetic stimulation of NTSR neurons. E. Overlay of fUSi and spiking activity for a colliculus recording. $r$ indicates correlation coefficient of the average activity on the probe and the corresponding pixels of the fUSi data. F. Dependence of correlation between fUSi and probe recordings on overall fUSi response strength. G. Average response (averaged across all optogenetic stimuli, backgroundsubtracted and normalized) of sorted units in six selected areas (Left; mean \pm std). Responses to optogenetic stimulation of 3 example cells for each area. The cell with the highest area under the curve (AUC) (second column), the medium AUC (third column) and the lowest AUC (fourth column) as well as the temporal fUSi response (last column). Numbers indicate AUC. H. Response strength to each optogenetic pulse sorted by AUC. $0 \%$ is background activity. I. Cumulative distribution of AUCs for each area. J. Responses to the 20 optogenetic pulses were clustered into 6 types. Average and STD of the normalized response strength for each cluster as well as average AUC (left) and \% of cells for each response type and area (Right).

SSC - superficial superior colliculus; dSC - deep superior colliculus; PAG - periaqueductal gray; LP - lateral posterior nucleus of the thalamus (pulvinar); CPc - caudate putamen; PPnT - posterior paralaminar nuclei of the thalamus. 
for a penetration (from Figure 6B) that passed through the cortex, the SC and periaqueductal gray (Figure 6D). Spikes were defined as local peaks of 4 times the standard deviation of the average activity before stimulation. Detected maxima during the $1 \mathrm{~ms}$ pulse itself were excluded since they were contaminated by electrical artifacts (see Methods). Clear responses to the 20 light pulses can be seen on the patch located in the superficial and deep colliculus (approximately electrodes 150 to 250, spanning 1000 um in depth). We aligned the histological slices including the probe tract with the Allen Brain Atlas, which allowed us to overlay the probe location with the fUSi data of the same coronal slice (Figure 6E). The spiking activity is indicated as a color-coded bar on top of the fUSi data. We averaged and normalized the fUSi data pooled from 6 brains along the Neuropixels probe track and compared it to the spiking activity. In this recording, we found a correlation coefficient between the fUSi and spiking signal of 0.83 (Figure 6E Right). We generally found a stronger correlation between the spiking activity and the fUSi signal in fUSi experiments with stronger responses (Figure 6F; correlation coefficient $r=0.69 ; n=26$ probe recordings).

\section{Optogenetic response patterns are different in the colliculus and downstream targets} Next, we asked how the optogenetic activation of neurons in the SC propagates through its direct and indirect output circuit elements. To this end we analyzed optogenetic responses in 6 different brain areas (Figure S5): 1) the superficial SC (sSC), 2) the deep SC (dSC), 3) The periaqueductal gray (PAG) which has previously been linked to aversive behaviors $\left.{ }^{50-52}, 4\right)$ the pulvinar (LP), which is a direct target of NTSR neurons, 5) The caudal caudoputamen (CPc), and 6) the posterior paralaminar nuclei of the thalamus (PPnT), which have not previously been linked to innate aversive behaviors but were strongly activated during NTSR stimulation (Figure 5H).

In all six areas, we found that the population of single neurons responded well to the optogenetic stimulation (Figure 6G first column). Similar to the raw spiking analysis, each area contained single neurons that responded well to all 20 pulses throughout the $1 \mathrm{~s}$ stimulation (Figure $6 \mathrm{G}$ second column) as well as cells that responded to the first stimulation only and others that were inhibited by further pulses (Figure 6G fourth column). We found that both the amplitude and the temporal changes in the spiking activity corresponded well to the fUSi signal recorded in the same brain areas (Figure 6G last column). When accounting for the delayed and slower blood response, the temporal profile of the fUSi signal and the probe recordings were similar for each of the tested areas. Areas with a stronger signal in the fUSi experiments showed a corresponding stronger spiking response (Figure 6G), and areas that showed a decrease in blood flow also showed a decrease in firing rate (Figure S6B and S6C).

To quantify the neural responses during the 20 optogenetic pulses, we calculated the mean, background-subtracted response of each responding neuron to the $40 \mathrm{~ms}$ after each pulse, normalized these 20 measurements to its maximal response and calculated the area under the curve (AUC) of those 20 values. An AUC of 1 indicates a cell that responds equally well to all 20 pulses, whereas negative AUC values indicate more inhibition than excitation. The resulting activity maps sorted by AUC indicate a different distribution of optogenetic responses in the 
different areas (Figure 6H; sSC $n=101$ units, dSC $n=392$, PAG $n=225$, LP $n=199, C P c$ $n=517$, PPnT $n=196$ ). We found a higher proportion of sustained responses (high AUC) in the superficial SC, more transient responses in the PPnT, CPc and periaqueductal gray, and a higher percentage of inhibited neurons in the pulvinar (Figure 6l). Subsequently, we then clustered the optogenetic responses into 6 types (Figure 6J). Neurons from the superficial SC were mostly in the more sustained clusters 1-3. Late-onset neurons (cluster 4) were almost absent in the $\mathrm{SC}$ but found in the other four multi-synaptic targets. Transient responses (cluster 5) are the dominant response type in the PPnT and inhibition was found in the pulvinar and PPnT (cluster 6). Taken together, these results show that optogenetic stimulation could be traced from the SC across several synapses. Response patterns were different at different stages downstream of NTSR neurons and the temporal profile of area-specific activity measured using spike recordings or fUSi corresponded well with each other.

\section{Visual responses downstream of NTSR neurons}

394 Activation of pulvinar-projecting neurons has been shown to induce arrest behavior 7,17 and we found arrest-like behavior when activating NTSR neurons (Figure 1). In addition, NTSR neurons respond well to visual stimuli mimicking attacking and over-head flying predators ${ }^{38}$ that induce aversive behaviors ${ }^{19,20}$. We thus tested whether neurons at different stages in the NTSR output circuitry that respond to optogenetic activation of NTSR neurons would also respond to behaviorally relevant visual stimuli. We found responses to a looming stimulus mimicking an attacking predator in optogenetically activated neurons in all tested brain areas (Figure 7A; sSC: 31 out of 57 optogenetically activated neurons, dSC: 76/139, PAG: 58/60, LP: 25/44, CPc: 59/109, PPnT: 14/50). Neurons showed different response properties including looming visual stimulus. These activation patterns were distributed differently in the six tested

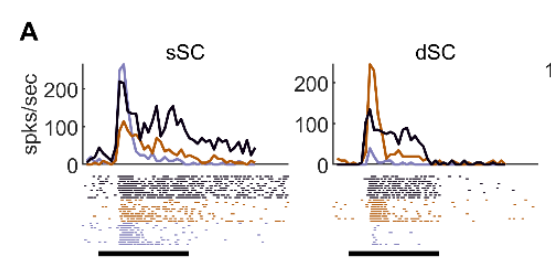

B

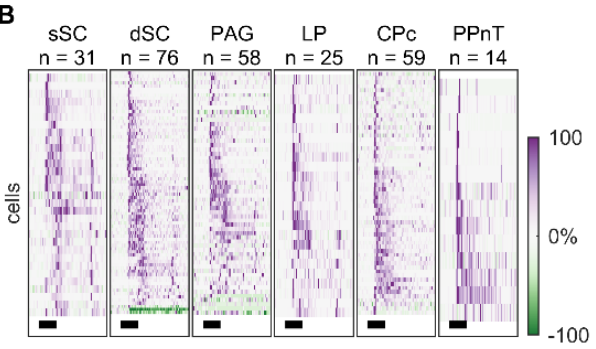

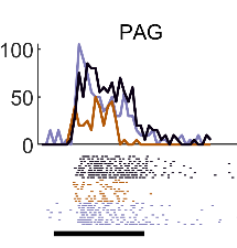

C
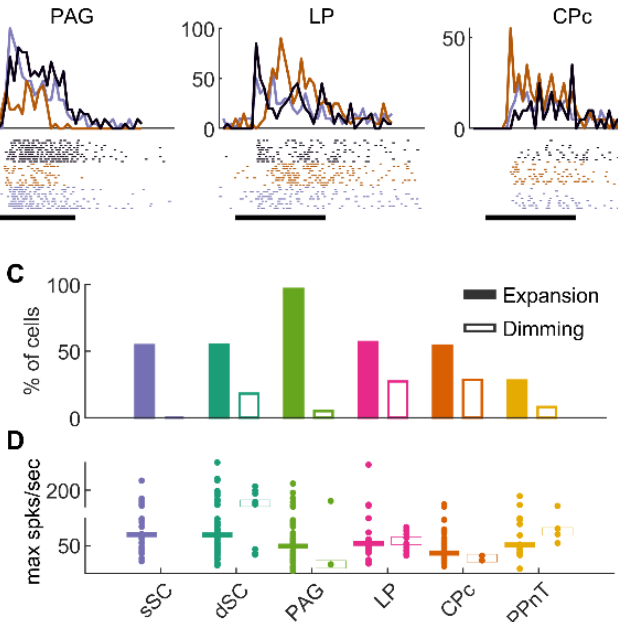

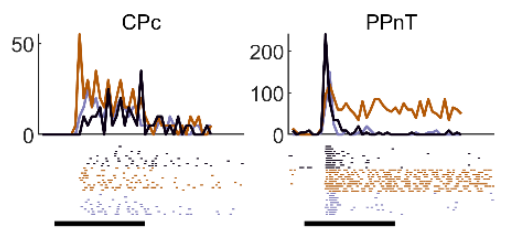

E

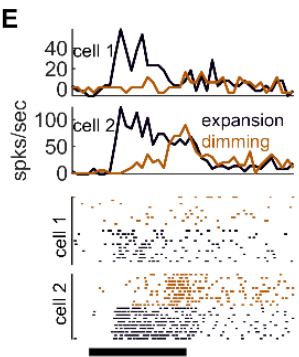

Figure 7. Visual responses in optogenetically activated cells of the NTSR-circuit. A. Example responses (raster and spikes/s) to a black looming stimulus for 3 cells with median response strength for each area. Black bar indicates time of looming $(0.315 \mathrm{~s})$. B. Normalized responses to a black looming disk of all optogenetically activated cells with looming responses in each area. C. Percentage of optogenetically activated cells with looming or dimming responses. $100 \%$ for looming/dimming for $\mathrm{SSC} n=57 / 6$, dSC $n=139 / 44$, PAG $n=60 / 59$, LP $n=44 / 44$, CPc $n=$ $109 / 7, \mathrm{PPnT} n=50 / 50$. D. Maximal response strength to expansion and dimming stimuli for each responding cell and their median per area. E. Example of cell with looming, but without dimming response (cell 1), and an example cell responding to both (cell 2). 
405

406

407

408

409

410

411

412

413

414

415

416

417

418

419

420

421

422

423

424

425

426

427

428

429

430

431

432

433

434

435

436

437

438

439

440

441

442

443

444

445

brain areas (Figure 7B). It is known that stimuli with similar properties as looming, but without ecological relevance, e.g. a dimming stimulus, do not elicit aversive behaviors ${ }^{19}$. In accordance with these behavioral findings, we could not detect responses to the dimming stimulus in optogenetically activated neurons of the SSC (Figure 7C). Neurons in further downstream areas sometimes responded to dimming stimuli at similar strength as for looming (Figure 7D and $7 \mathrm{E}$ ). However, in all areas, fewer neurons responded to dimming stimuli as compared to looming stimuli (sSC: 0 out of 6 optogenetically responding cells, dSC: 8/44, PAG: 3/59, LP: 12/44, CPc: 2/7, PPnT: 4/50). We found no dimming responses in the sSC even when including units that did not respond to optogenetic stimulation and similar higher percentages of dimming responses in the LP, CPc, and PPnT (Figure S6). These data show that ecologically relevant visual information is present throughout the multi-synaptic downstream networks of the colliculus that is revealed during opto-fUSi imaging.

\section{Inhibition of PPnT facilitates habituation to repeated stimulation of NTSR neurons.}

The PPnT has not been previously shown to participate in collicular driven behaviors. Our fUSi data showed that it is consistently activated in response to the stimulation of CAMKII, NTSR and PV neurons of the colliculus (Figure 5). We corroborated that neurons in the PPnT respond to both optogenetic stimulation of NTSR neurons and visual stimuli (Figure 6 and 7). To investigate its role in defensive behaviors, we chemogenetically suppressed activity of its neurons while optogenetically stimulating NTSR neurons in the SC. We injected an AAV coding for the inhibitory DREADDs hM4D(qi) under human synuclein 1 promoter into the PPnT of NTSRxChR2 mice (Figure 8A-B). The same optogenetic stimulation protocol as in previous experiments was used to activate ChR2 in NTSR neurons. We tested mice in the open field arena (Figure $1 \mathrm{~A})$ and optogenetically stimulated $(20 \mathrm{~Hz}$ for $1 \mathrm{~s})$ as the mouse crossed the center of the arena. We conducted five experimental sessions, separated by at least 2 days (Figure 8A). To inhibit the PPnT, clozapine N-oxide (CNO) was injected intra-peritoneally 2030 minutes before the beginning of the second session.

We found that inhibition of PPnT increased the variability in the responsiveness of mice to the optical stimulation (Figure $8 \mathrm{C}-\mathrm{E}$ ). This variability manifested as a decrease in the probability that arrest would be triggered by the optogenetic stimulus after a CNO injection (Figure 8F, Movie S17 and Movie S18). During subsequent sessions mice did not regain the lost behavioral response (Figure $8 \mathrm{G}$ ) and tended to maintain a higher speed than controls during the stimulation periods (Figure $8 \mathrm{H}$ ).

To investigate the relationship between stopping behavior and the specific location of hM4D expression in the PPnT, we examined the correlation between stopping probability in Session 2 (when CNO was first administered) and the coordinates of the center of expression (Figure S7A-C), or the antero-posterior spread of the expression (Figure S7D). Linear regression revealed weak correlations with the AP, ML, DV planes and extent of expression (Pearson coefficient $r=0.112,0.245,-0.293$ and -0.297 respectively). Additionally, we compared the stopping probability of animals with and without DREADD expression in the different areas included in the PPnT (Figure S7E). All the examined mice had expression in the POL. The PoT was present in most animals $(n=13 / 17)$, but the presence or absence of expression in this area 
A

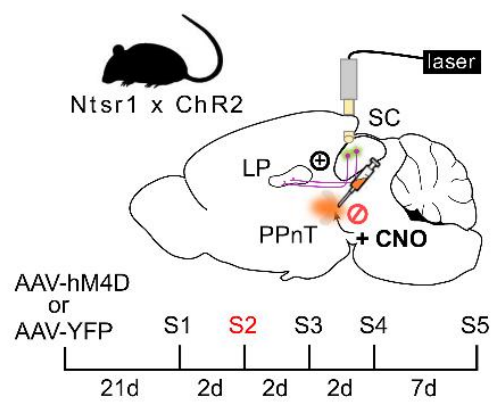

B

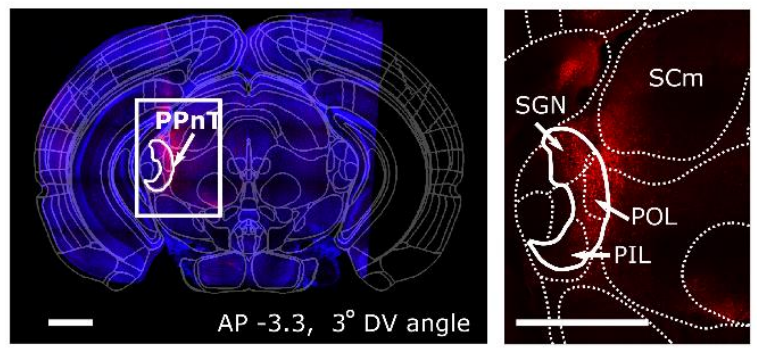

C
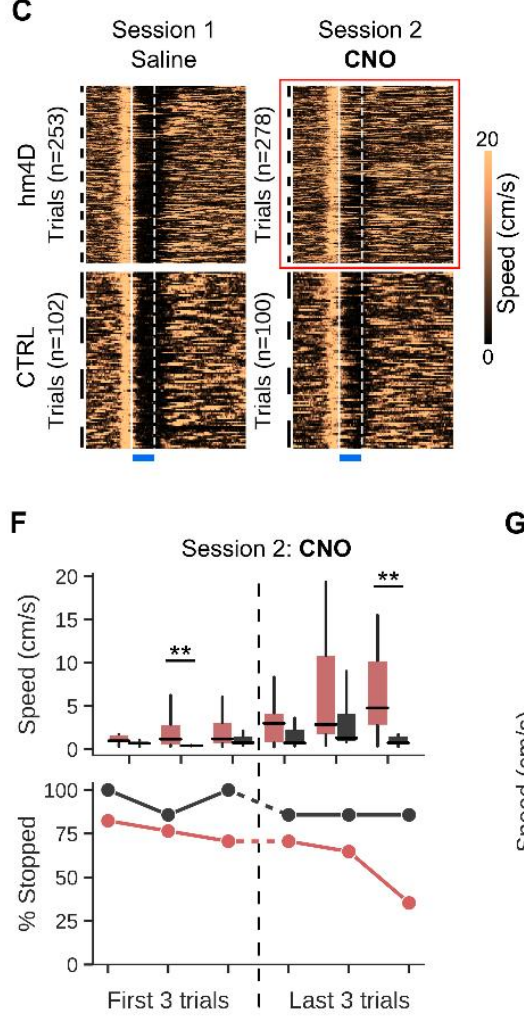

D
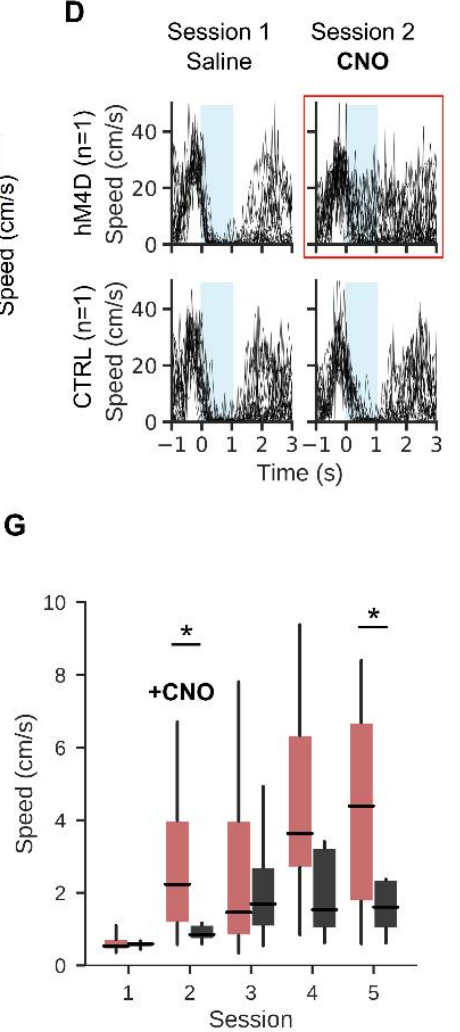

E

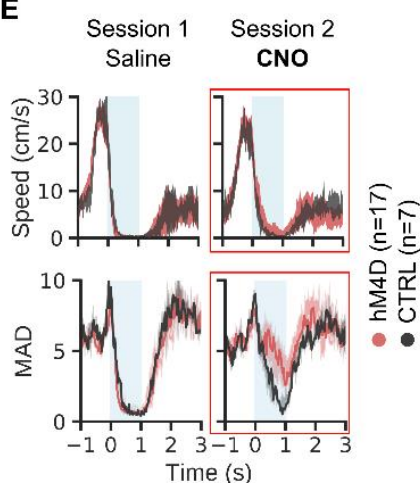

H

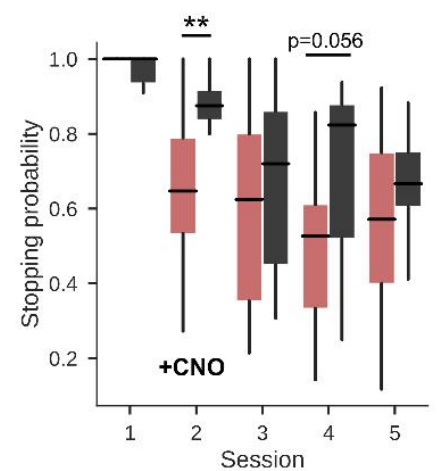

Figure 8. PPnT inhibition facilitates habituation to repeated optogenetic stimulation. A. Experimental paradigm B. Expression of AAV2-hSyn-hM4D(Gi)-mCherry (red) in the PPnT of a NTSR x Chr2 mouse. Left, coronal brain section aligned to Allen Mouse brain atlas. White rectangle indicates injection site and corresponds to right, zoomed in area. Scale bar $1 \mathrm{~mm}$. C. Speed traces of NTSR $\times$ Chr2 mice injected with AAV-hM4D (Top row, n=17) and controls ( $\mathrm{n}=7$, Bottom row), injected with AAV-YFP for session 1 (saline) and session 2 (CNO). Black lines delineate trials belonging to single animals. White lines delineate onset and offset of optogenetic stimulus. D. Speed traces from all trials in session 1 (Left) and 2 (Right) from one example animal from each group. E. Top row: median speed traces from all hM4D (red) and CTRL (gray) mice during sessions 1 and 2. Bottom row: mean absolute deviation of the speed for hM4D (red) and CTRL (gray) mice. F. Median speed (Top row) and stopping probability (Bottom row) during the one second stimulation of hM4D (red) and CTRL (gray) mice, within the session where CNO was administered. G. Median speed of hM4D (red) and CTRL (gray) mice, during the $1 \mathrm{~s}$ stimulations across sessions $\mathbf{H}$. Stopping probability of hM4D (red) and CTRL (gray) mice, during the $1 \mathrm{~s}$ stimulations across sessions. Box plots indicate median, interquartile range, and 5 th to 95 th percentiles of the distribution. ${ }^{*} p<0.05 ;{ }^{* *} p<0.01$. 
448 Interestingly, expression in the PIL and SGN was observed in approximately $50 \%$ of the animals (PIL: $n=9 / 17$; SGN: $n=8 / 17$ ) and rather than causing the behavioral attenuation, expression in these areas seemed to interfere with the effect, increasing stopping probability (Mann-Whitney U-test; PIL: $p=0.037$; SGN: $p=0.042$ ). We also observed viral expression in the most caudal part of the LP in several animals $(n=8 / 17)$, but its presence did not correlate with a reduced stopping probability (Mann-Whitney U-test; $p=0.168$, Figure S8F). Overall, these results suggest that the inhibition of the PPnT, most likely through the POL, facilitates habituation to the repeated activation of collicular NTSR neurons.

\section{Discussion}

458

In this study, we combined fUSi with optogenetics to reveal the whole-brain neuronal networks that link individual cell-types of the SC with a triggered behavior. We show here that the mouse colliculus distributes information encoded in specific cell-types through distinct networks that share a set of common nodes. Three principals have emerged from this work. First, the spatial and temporal activity patterns evoked by each cell-type are distinct from each other. Second, the observed differences (or similarities) in behavior could not be explained by the activity in any single brain region but appear to be the consequence of distributed activity across many, predominately, subcortical brain areas. Third, while fUSi imaging revealed activation of known downstream targets of each cell-type, it also revealed activity in a set of areas previously not considered as part of these behavioral networks. This allowed us to uncover a putative role of one of these novel targets, the PPnT, in habituation. Direct measurements of neural activity using silicon probes demonstrate a strong correspondence in both space and time between the fUSi signal and mean firing rate in each brain region. Using probe recordings, we also found responses to ecologically relevant visual stimuli in brain regions identified as part of the functional network. Taken together, these results support the notion that in the SC individual cell-types trigger distinct behaviors, not via single dedicated pathways, but instead via distinct brain-wide networks that share a common set of nodes.

474 Collicular cell-types activate different, partially overlapping downstream networks.

475 A variety of previous lines of evidence are consistent with our finding that activation of different 476 cell-types of the SC and their output pathways leads to the broad yet restricted propagation of information across the brain. We found that each collicular cell-type relayed information through a different downstream network, that converge in a few key nodes (Figure 4 and 5). Our fUSi experiments show that activation of each cell-type modulated the neural activity of at least 68 and up to 193 brain areas. Among the pathways activated were a set of areas that are consistent with known output pathways of the SC and that have been identified to trigger freezing and escape behaviors ${ }^{6,7,10,12-14,50}$. Here we demonstrate that activation of the same neural populations results in neural activity in a much larger than expected set of downstream areas. This extensive dissemination of information is likely due in part to recurrent connectivity within the SC ${ }^{12,16}$, as well as recurrent feedback loops with, for example, deeper layers of the SC, PAG, thalamus and PBG ${ }^{14}$. While we do observe the previously reported activity in specific 
nuclei, brain-wide fUSi allowed us to observe functional networks downstream of cell-types in the SC across the most of the brain.

489 Conversely, the brain-wide activity we observed shows a higher degree of specificity than we 490 would predict from meso-scale maps of area-to-area connectivity ${ }^{53}$. If, for example, we assume each brain area, like the retinal recipient layers of the SC, projects to at least 6 downstream structures we only need 3 synapses to modulate 216 brain areas. In many cases this underestimates the number of projections a brain area makes. The retina sends projections to approximately 40 targets ${ }^{54}$, while the primary visual cortex innervates at least 18 cortical and subcortical areas ${ }^{55}$. We saw our maximal spread of activity when stimulating CAMKII neurons, which modulated 246 areas during the early phase, while NTSR neurons modulated 146 areas. This restriction in the extent of dissemination is likely due to the cell-type specific connection made in the SC and other brain regions. In the SC, distinct output pathways are known to selectively sample retinal inputs and project to selected downstream areas ${ }^{13,56}$. In addition, outputs of the SC to the LP have been shown to be relayed to a narrow set of downstream targets in the visual cortex and amygdala ${ }^{17,48}$. This cell-type and pathway specific relay of information is a common feature of several brain structures investigated, including the visual cortex, amygdala and VTA ${ }^{55,57,58 .}$ How the SC routes the cell-type specific information to evoke different behaviors corresponded well with the observed similarities and differences in the network activity. We observed that activation of CAMKII and NTSR neurons each resulted in an interruption of locomotor activity, which was reflected in the similarity in the brain-wide activity evoked by each cell population (Figure 4). While activating CAMKII and NTSR neurons each interrupted locomotor activity, activating CAMKII neurons resulted in prolonged periods of immobility as compared to NTSR neurons (Figure 1), which corresponded well with the observed differences in the temporal response profiles (Figure 5). For example, in comparison to NTSR, stimulation of CAMKII neurons evoked prolonged activity in areas including the premammillary nucleus of the hypothalamus (PMH) and the superior central nucleus raphe (CS). In addition, we demonstrated that activation of PV and GAD2 neurons facilitated active avoidance and orienting movements, respectively. In comparison to CAMKII and NTSR, activation of PV and GAD2 neurons was characterized by increases in the activity of cerebellar areas like CENT2 and IP. To further discuss how different behaviors are mediated, we focus below on three brain areas: the subthalamic nucleus (STN), the cuneiform nucleus (CUN) and midline thalamus nuclei.

521 First, activating either CAMKII or NTSR neurons resulted in increase in the activity of the 522 subthalamic nucleus (STN) - a region involved in the interruption of ongoing behaviors ${ }^{59,60}$. In 523 contrast, the activity of STN was suppressed or not detectable after stimulation of PV and 524 GAD2 cell-types, where locomotion was not interrupted. This suggests that the STN is either 525 activated to pause ongoing behaviors or silenced to promote escape strategies. Second, we 526 found distinct temporal responses in the CUN across mouse lines. The CUN has been shown 527 to trigger freezing and escape ${ }^{44}$, participate in the initiation and control of locomotion ${ }^{61,62}$ and 
modulate cardiovascular response ${ }^{63}$. We observed fast, transient responses in animals with freezing-like behaviors (CAMKII, NTSR) and slow, more sustained responses, in animals with 530 continuous locomotion (PV, GAD2). It is therefore plausible that different neuronal 531 subpopulations of the CUN are functionally connected with different collicular cell-types to play 532 various roles in defensive behaviors. Third, we demonstrated that the nuclei of ventral midline 533 thalamus (RE and $\mathrm{Xi}$ ), which play a role in decision making when exposed to threat ${ }^{64}$, are 534 modulated by both PV and CAMKII neurons but in opposite direction, with sustained activation and inhibition, respectively (Figure 5). Taken together our results suggest that opto-fUSi is a reliable method for studying how the cell-type specific information is disseminated from the SC across the brain to trigger behavior.

\section{Opto-fUSi reveals new players in aversive behavior driven by the SC}

539 In our experiments, we observed areas consistently activated that have not previously been reported to be involved in mediating visually guided aversive behaviors. Precise circuit dissections have highlighted three major pathways that pass information about visual threat from the SC to downstream areas. These cell-type specific pathways include projections to

543 the amygdala through the LP or the PBG ${ }^{6,7,17}$; and projections to the APAG ${ }^{50}$. These dissections have led to an atomistic understanding of how the SC mediates aversive behaviors. Here we observed activity in many areas across the brain and four of them captured our attention, namely: the caudal part of caudoputamen (CPc), postrhinal visual area (VISpor), posterior lateral part of the midbrain reticular formation (MRNpl) and PPnT (Figure 5). Some of these areas have been implicated in modulation of visual behavior ${ }^{65-68}$. The PPnT stood out during visual inspection of activity maps of the brain, as it was both reliably activated upon optogenetic stimulation of NTSR neurons of the SC and during the presentation of visual stimuli. The PPnT is known for its role in associative learning during auditory fear conditioning and in mediating fear discrimination and extinction ${ }^{69,70}$. Our experiments revealed that this group of areas play a similar role in behaviors triggered by the SC by acting to suppress habituation. Our results are consistent with the proposed role of PPnT in fear extinction and suggest that PPnT is part of the pathway downstream of the SC and is involved in mediating behaviors triggered from the SC. These results highlight the power of combining optogenetic manipulations with brain-wide observation of neuronal activity, which provides a method to identify the brain-wide networks involved and thus the design experiments that allow us to build a more complete picture of how defensive behaviors are controlled.

\section{Optogenetics and the relationship between fUSi and neural activity}

561 One of the key questions for fUSi imaging is the degree to which it faithfully represents the 562 underlying neural activity. In the context of $\mathrm{FMRI}$, while it is generally accepted that the 563 measured blood oxygen-level dependent (BOLD) signal changes are associated with neuronal 564 activity ${ }^{71}$; how and where the BOLD signal reliably represents the spiking activity of individual 565 neurons still remains an open question ${ }^{72}$. Like fMRI, fUSi also relies on the indirect 566 measurement of neural activity through hemodynamic changes, in this case, in cerebral blood 567 volume. Recently, it has been shown that fUS signals, can reliably represent both increases 568 and decreases in local neuronal activity ${ }^{28,33}$. We provide additional evidence that the changes 
569 in blood volume detected by the fUSi are consistent with the local changes in neuronal activity

570 in a number of different brain regions including parts of the cortex, striatum, hippocampus,

571 thalamus and midbrain (Figure 6 and Figure S6).

572 Light delivery into the brain, similar to our optogenetic activation, has been reported to cause

573 local temperature changes and arterial vasodilation in naïve mice and rats that can cause

574 artefactual signals both in opto-fMRI and opto-fUSi ${ }^{73,74}$. To minimize such potential effects, in

575 our experiments, we used shorter and lower light intensity stimuli (0.3-0.4 mW, 2ms pulses,

$57620-50 \mathrm{~Hz}, 1 \mathrm{~s})$ than the energy threshold calculated ( $<1 \mathrm{~mW}, 20 \mathrm{~ms}$ pulses, $20 \mathrm{~Hz}, 2 \mathrm{~s})$ by

577 Rungta et al. 2017. We did not observe any hemodynamic signal in control experiments using

578 naïve mice in these conditions, indicating that the results reported in this study are driven by

579 the activation of collicular cell-types. In addition, we found that the magnitude, sign, and time

580 course of the fUSi signal corresponded well with the local spiking activity measured in the

581 same area of the brain and in response to the same stimuli.

582 Brain-wide mapping of function using Opto-fUSi

583 Understanding the neural basis of defensive behaviors is relevant not only because these

584 behaviors are important for survival, but also because their dysregulation may contribute to

585 anxiety and post-traumatic disorders. To accomplish this, it is necessary to have a holistic

586 understanding of how the nervous system integrates information to guide appropriate

587 behaviors, as well as to know how molecularly defined components of the nervous system

588 contribute to this neuronal activity. Here, we have presented a set of experiments that highlight

589 how combining optogenetics with fUSi can bridge this divide, enabling both the manipulation

590 of targeted components of the nervous system and the simultaneous monitoring of brain-wide

591 activity.

592 Various methods enable us to look at activity across large parts of the brain, including fMRI, 593 wide-field calcium imaging, large-scale electrophysiology, and genetic markers of neural

594 activity. However, fMRI suffers from low temporal and spatial resolution ${ }^{75,76}$. Cortical wide 595 calcium imaging and large-scale probe recordings have excellent temporal and spatial 596 resolution but only allow for measurements that are limited to cortex, in the case of calcium 597 imaging, or in thin columns near the electrode tract for silicon probe recordings $3,77,78$. Finally, 598 genetic markers that putatively report elevated levels of neural activity (e.g. cfos or CaMPARI) 599 provide single cell resolution across the whole brain but have poor temporal resolution and 600 their activation is difficult to interpret ${ }^{79,80}$.

601 fUSi enables access to the whole brain at a spatial and temporal resolution $\left(\sim 100 \mu \mathrm{m}^{3}, 0.1 \mathrm{~s}\right)$ 602 that allowed us to map the neural network activated by defined cell populations of the SC 603 across a larger portion of the brain. To our knowledge, this is the first time a comprehensive 604 brain-wide mapping has been done at this spatiotemporal resolution of the circuits involved in 605 innate defensive behaviors of mice. Previous experiments in humans and primates have 606 provided evidence of the involvement of structures such as the LC, the PAG, the SC, the visual 607 thalamus, the amygdala, the insular cortex and PFC ${ }^{23-25,81-83}$. In rodents, molecular (cfos) 608 functional brain maps have implicated a few additional thalamic, hypothalamic and cerebellar 
609 areas in various fearful conditions ${ }^{64,84-88}$. We analyzed the activity of 264 areas across the 610 mouse brain. Our results indicate that the neural pathways involved in mediating and 611 modulating the behavioral responses to activation of the SC stimuli is far more complex that 612 previously reported. We believe combining fUSi with targeted cell-type manipulations and 613 natural stimuli will allow us to understand how different brain regions act in concert to guide 614 defensive behaviors under a variety of conditions.

615 


\section{ACKNOWLEDGMENTS}

617 This work was supported by the FWO (G094616N to KF, G091719N to KF and AU, MEDI$618 \mathrm{RESCU}_{2}$-AKUL/17/049 and $1197818 \mathrm{~N}$ to $\mathrm{AU}, 1197818 \mathrm{~N} / 1197820 \mathrm{~N}$ to ASD, $61911 \mathrm{C} 5119 \mathrm{~N} / 11 \mathrm{C} 5121 \mathrm{~N}$ to $\mathrm{AC}$ and $12 \mathrm{~S} 7920 \mathrm{~N}$ to KR); The Leducq Foundation (15CVD02 to AU); 620 the European Union's Horizon 2020 research and innovation programme under the Marie 621 Skłodowska-Curie grant agreement No 665501 (12S7917N to KR); A Master Mind Scholarship 622 (F200075 to DL).

\section{AUTHOR CONTRIBUTIONS}

624 Arnau Sans Dublanc, Conceptualization, Data curation, Formal analysis, Funding acquisition, 625 Validation, Investigation, Visualization, Methodology, Writing—original draft, Writing—review 626 and editing; Anna Chrzanowska, Conceptualization, Data curation, Funding acquisition, 627 Investigation, Visualization, Methodology, Writing—original draft, Writing—review and editing; 628 Katja Reinhard, Conceptualization, Data curation, Supervision, Funding acquisition, 629 Investigation, Visualization, Methodology, Writing—original draft, Writing—review and editing; 630 Dani Lemmon, Data curation, Funding acquisition, Investigation, Visualization; Gabriel 631 Montaldo, Conceptualization, Methodology, Software, Supervision, Writing-review and 632 editing; Alan Urban, Conceptualization, Funding acquisition, Methodology, Software, 633 Supervision, Writing-review and editing; Karl Farrow, Conceptualization, Software, Formal 634 analysis, Supervision, Funding acquisition, Investigation, Visualization, Methodology, Writing635 original draft, Project administration, Writing—review and editing. 
637 1. Zeng, H. \& Sanes, J. R. Neuronal cell-type classification: challenges, opportunities and 638 the path forward. Nat. Rev. Neurosci. 18, 530-546 (2017).

639 2. Stringer, C. et al. Spontaneous behaviors drive multidimensional, brainwide activity.

3. Steinmetz, N. A., Zatka-Haas, P., Carandini, M. \& Harris, K. D. Distributed coding of Science (80-. ). 364, (2019).

4. Ahrens, M. B. et al. Brain-wide neuronal dynamics during motor adaptation in zebrafish.

5. Aimon, S. et al. Fast near-whole-brain imaging in adult Drosophila during responses to

6. Shang, C. et al. A parvalbumin-positive excitatory visual pathway to trigger fear

7. Shang, C. et al. Divergent midbrain circuits orchestrate escape and freezing responses

8. Hoy, J. L., Bishop, H. I. \& Niell, C. M. Defined Cell Types in Superior Colliculus Make

9. Masullo, L. et al. Genetically Defined Functional Modules for Spatial Orienting in the Distinct Contributions to Prey Capture Behavior in the Mouse. Curr. Biol. 29, 41304138.e5 (2019).

Mouse Superior Colliculus. Curr. Biol. 29, 2892-2904.e8 (2019).

10. Zhang, Z. et al. Superior Colliculus GABAergic Neurons Are Essential for Acute Dark Induction of Wakefulness in Mice. Curr. Biol. 29, 637-644.e3 (2019).

11. Ellis, E. M., Gauvain, G., Sivyer, B. \& Murphy, G. J. Shared and distinct retinal input to the mouse superior colliculus and dorsal lateral geniculate nucleus. J. Neurophysiol. 116, 602-610 (2016).

12. Gale, S. D. \& Murphy, G. J. Distinct cell types in the superficial superior colliculus project to the dorsal lateral geniculate and lateral posterior thalamic nuclei. J. Neurophysiol. 120, 1286-1292 (2018).

13. Gale, S. D. \& Murphy, G. J. Distinct representation and distribution of visual information by specific cell types in mouse superficial superior colliculus. J. Neurosci. 34, 1345813471 (2014).

14. May, P. J. The mammalian superior colliculus: Laminar structure and connections. Progress in Brain Research 151, 321-378 (2006).

15. Inayat, S. et al. Neurons in the Most Superficial Lamina of the Mouse Superior Colliculus Are Highly Selective for Stimulus Direction. J. Neurosci. 35, 7992-8003 (2015).

16. Whyland, K. L., Slusarczyk, A. S. \& Bickford, M. E. GABAergic cell types in the superficial layers of the mouse superior colliculus. J. Comp. Neurol. 528, 308-320 (2020).

17. Wei, P. et al. Processing of visually evoked innate fear by a non-canonical thalamic pathway. Nat. Commun. 6, 6756 (2015).

18. Zhang, Z. et al. Superior Colliculus GABAergic Neurons Are Essential for Acute Dark Induction of Wakefulness in Mice. Curr. Biol. 29, 637-644.e3 (2019). 
701

702

703

704

705

706

707

708

709

710

711

712

713

714

715

716

717

718

19. Yilmaz, M. \& Meister, M. Rapid innate defensive responses of mice to looming visual stimuli. Curr. Biol. 23, 2011-2015 (2013).

20. De Franceschi, G., Vivattanasarn, T., Saleem, A. B. \& Solomon, S. G. Vision Guides Selection of Freeze or Flight Defense Strategies in Mice. Curr. Biol. 26, 2150-2154 (2016).

21. Lee, H. J. et al. Activation of Direct and Indirect Pathway Medium Spiny Neurons Drives Distinct Brain-wide Responses. Neuron 91, 412-424 (2016).

22. Nakamura, Y. et al. fMRI detects bilateral brain network activation following unilateral chemogenetic activation of direct striatal projection neurons. Neuroimage 220, 117079 (2020).

23. Almeida, I., Soares, S. C. \& Castelo-Branco, M. The distinct role of the amygdala, superior colliculus and pulvinar in processing of central and peripheral snakes. PLoS One 10, (2015).

24. Liddell, B. J. et al. A direct brainstem-amygdala-cortical 'alarm' system for subliminal signals of fear. Neuroimage 24, 235-243 (2005).

25. Terpou, B. A. et al. The Innate Alarm System and Subliminal Threat Presentation in Posttraumatic Stress Disorder: Neuroimaging of the Midbrain and Cerebellum. Chronic Stress 3, 247054701882149 (2019).

26. Bernal-Casas, D., Lee, H. J., Weitz, A. J. \& Lee, J. H. Studying Brain Circuit Function with Dynamic Causal Modeling for Optogenetic fMRI. Neuron 93, 522-532.e5 (2017).

27. Mace, E. et al. Functional ultrasound imaging of the brain: theory and basic principles. IEEE Trans. Ultrason. Ferroelectr. Freq. Control 60, 492-506 (2013).

28. Macé, É. et al. Whole-Brain Functional Ultrasound Imaging Reveals Brain Modules for Visuomotor Integration. Neuron 100, 1241-1251.e7 (2018).

29. Urban, A. et al. Chronic assessment of cerebral hemodynamics during rat forepaw electrical stimulation using functional ultrasound imaging. Neuroimage 101, 138-149 (2014).

30. Rabut, C. et al. 4D functional ultrasound imaging of whole-brain activity in rodents. Nat. Methods 16, 994-997 (2019).

31. Brunner, C. et al. A platform for brain-wide functional ultrasound imaging and analysis of circuit dynamics in behaving mice. bioRxiv (2020). doi:10.1101/2020.04.10.035436

32. Macé, E. et al. Functional ultrasound imaging of the brain. Nat Methods 8, 662-664 (2011).

33. Aydin, A.-K. et al. Transfer functions linking neural calcium to single voxel functional ultrasound signal. Nat. Commun. 11, 2954 (2020).

34. Taniguchi, H. et al. A resource of Cre driver lines for genetic targeting of GABAergic neurons in cerebral cortex. Neuron 71, 995-1013 (2011).

35. Madisen, L. et al. A toolbox of Cre-dependent optogenetic transgenic mice for lightinduced activation and silencing. Nat. Neurosci. 15, 793-802 (2012).

36. Gerfen, C. R., Paletzki, R. \& Heintz, N. GENSAT BAC cre-recombinase driver lines to study the functional organization of cerebral cortical and basal ganglia circuits. Neuron 80, 1368-1383 (2013). 
37. Madisen, L. et al. A robust and high-throughput Cre reporting and characterization system for the whole mouse brain. Nat. Neurosci. 13, 133-140 (2010).

38. Gale, S. D. \& Murphy, G. J. Active Dendritic Properties and Local Inhibitory Input Enable Selectivity for Object Motion in Mouse Superior Colliculus Neurons. J. Neurosci. 36, 9111-23 (2016).

39. Rubin, J. M. et al. Fractional moving blood volume: estimation with power Doppler US. Radiology 197, 183-190 (1995).

40. Rubin, J. M., Bude, R. O., Carson, P. L., Bree, R. L. \& Adler, R. S. Power Doppler US: a potentially useful alternative to mean frequency-based color Doppler US. Radiology 190, 853-856 (1994).

41. Lein, E. S. et al. Genome-wide atlas of gene expression in the adult mouse brain. Nature 445, 168-176 (2007).

42. Sparks, D. L. The brainstem control of saccadic eye movements. Nat. Rev. Neurosci. 3, 952-964 (2002).

43. Isa, T. \& Sasaki, S. Brainstem control of head movements during orienting; Organization of the premotor circuits. Prog. Neurobiol. 66, 205-241 (2002).

44. Dean, P., Redgrave, P. \& Westby, G. W. M. Event or emergency? Two response systems in the mammalian superior colliculus. Trends Neurosci. 12, 137-147 (1989).

45. Herkenham, M. New Perspectives on the Organization and Evolution of Nonspecific Thalamocortical Projections. in 403-445 (Springer, Boston, MA, 1986). doi:10.1007/978-1-4613-2149-1_11

46. Harting, J. K., Updyke, B. V. \& Van Lieshout, D. P. Striatal projections from the cat visual thalamus. Eur. J. Neurosci. 14, 893-896 (2001).

47. Takahashi, T. The organization of the lateral thalamus of the hooded rat. J. Comp. Neurol. 231, 281-309 (1985).

48. Beltramo, R. \& Scanziani, M. A collicular visual cortex: Neocortical space for an ancient midbrain visual structure. Science (80-. ). 363, 64-69 (2019).

49. Bennett, C. et al. Higher-Order Thalamic Circuits Channel Parallel Streams of Visual Information in Mice. Neuron 102, 477-492.e5 (2019).

50. Evans, D. A. et al. A synaptic threshold mechanism for computing escape decisions. Nature 558, 590-594 (2018).

51. Watson, T. C., Cerminara, N. L., Lumb, B. M. \& Apps, R. Neural correlates of fear in the periaqueductal gray. J. Neurosci. 36, 12707-12719 (2016).

52. Deng, H., Xiao, X. \& Wang, Z. Periaqueductal gray neuronal activities underlie different aspects of defensive behaviors. J. Neurosci. 36, 7580-7588 (2016).

53. Oh, S. W. et al. A mesoscale connectome of the mouse brain. Nature 508, 207-214 (2014).

54. Martersteck, E. M. et al. Diverse Central Projection Patterns of Retinal Ganglion Cells. Cell Rep. 18, 2058-2072 (2017).

55. Han, Y. et al. The logic of single-cell projections from visual cortex. Nature 556, 51-56 (2018). 
56. Reinhard, K. et al. A projection specific logic to sampling visual inputs in mouse superior colliculus. Elife 8, (2019).

57. Beier, K. T. et al. Topological Organization of Ventral Tegmental Area Connectivity Revealed by Viral-Genetic Dissection of Input-Output Relations. Cell Rep. 26, 159167.e6 (2019).

58. Fadok, J. P. et al. A competitive inhibitory circuit for selection of active and passive fear responses. Nature 542, 96-99 (2017).

59. Aron, A. R. \& Poldrack, R. A. Cortical and subcortical contributions to stop signal response inhibition: Role of the subthalamic nucleus. J. Neurosci. 26, 2424-2433 (2006).

60. Fife, K. H. et al. Causal role for the subthalamic nucleus in interrupting behavior. Elife 6, $1-13(2017)$.

61. Capelli, P., Pivetta, C., Esposito, M. S. \& Arber, S. Locomotor speed control circuits in the caudal brainstem. Nature 551, 373-377 (2017).

62. Mori, S., Sakamoto, T., Ohta, Y., Takakusaki, K. \& Matsuyama, K. Site-specific postural and locomotor changes evoked in awake, freely moving intact cats by stimulating the brainstem. Brain Res. 505, 66-74 (1989).

63. Korte, S. M., Jaarsma, D., Luiten, P. G. M. \& Bohus, B. Mesencephalic cuneiform nucleus and its ascending and descending projections serve stress-related cardiovascular responses in the rat. J. Auton. Nerv. Syst. 41, 157-176 (1992).

64. Salay, L. D., Ishiko, N. \& Huberman, A. D. A midline thalamic circuit determines reactions to visual threat. Nature 557, 183-189 (2018).

65. Reig, R. \& Silberberg, G. Article Multisensory Integration in the Mouse Striatum. Neuron 83, 1200-1212 (2014).

66. Nagy, A., Eördegh, G., Norita, M. \& Benedek, G. Visual receptive field properties of neurons in the caudate nucleus. Eur. J. Neurosci. 18, 449-452 (2003).

67. Schulz, J. M. et al. Short-latency activation of striatal spiny neurons via subcortical visual pathways. J. Neurosci. 29, 6336-6347 (2009).

68. Wilson, J. S., Hull, C. D. \& Buchwald, N. A. Intracellular studies of the convergence of sensory input on caudate neurons of cat. Brain Res. 270, 197-208 (1983).

69. Grosso, A., Santoni, G., Manassero, E., Renna, A. \& Sacchetti, B. A neuronal basis for fear discrimination in the lateral amygdala. Nat. Commun. 9, (2018).

70. Gross, C. T. \& Canteras, N. S. The many paths to fear. Nature Reviews Neuroscience 13, 651-658 (2012).

71. Logothetis, N. K. \& Wandell, B. A. Interpreting the BOLD signal. Annu. Rev. Physiol. 66, 735-769 (2004).

72. Ekstrom, A. How and when the fMRI BOLD signal relates to underlying neural activity: The danger in dissociation. Brain Res. Rev. 62, 233-244 (2010).

73. Rungta, R. L., Osmanski, B. F., Boido, D., Tanter, M. \& Charpak, S. Light controls cerebral blood flow in naive animals. Nat. Commun. 8, (2017).

74. Christie, I. N. et al. FMRI response to blue light delivery in the naïve brain: Implications 
for combined optogenetic fMRI studies. Neuroimage 66, 634-641 (2013).

75. Jung, W. B., Shim, H. J. \& Kim, S. G. Mouse BOLD fMRI at ultrahigh field detects somatosensory networks including thalamic nuclei. Neuroimage 195, 203-214 (2019).

76. Lewis, L. D., Setsompop, K., Rosen, B. R. \& Polimeni, J. R. Fast fMRI can detect Oscillatory neural activity in humans. Proc. Natl. Acad. Sci. U. S. A. 113, E6679-E6685 (2016).

77. Allen, W. E. et al. Global Representations of Goal-Directed Behavior in Distinct Cell Types of Mouse Neocortex. Neuron 94, 891-907.e6 (2017).

78. Omlor, W. et al. Context-dependent limb movement encoding in neuronal populations of motor cortex. Nat. Commun. 10, (2019).

79. Moeyaert, B. et al. Improved methods for marking active neuron populations. Nat. Commun. 9, 1-12 (2018).

80. Morgan, J. I. \& Curran, T. Stimulus-transcription coupling in the nervous system: Involvement of the inducible proto-oncogenes fos and jun. Annual Review of Neuroscience 14, 421-451 (1991).

81. Koizumi, A. et al. Threat anticipation in pulvinar and in superficial layers of primary visual cortex (V1). Evidence from Layer-Specific Ultra-High Field 7T fMRI. eNeuro 6, (2019).

82. Shiba, Y. et al. Converging Prefronto-Insula-Amygdala Pathways in Negative Emotion Regulation in Marmoset Monkeys. Biol. Psychiatry 82, 895-903 (2017).

83. Morris, J. S., DeBonis, M. \& Dolan, R. J. Human amygdala responses to fearful eyes. Neuroimage 17, 214-222 (2002).

84. Chou, X. L. et al. Inhibitory gain modulation of defense behaviors by zona incerta. Nat. Commun. 9, (2018).

85. Mendes-Gomes, J. et al. Defensive behaviors and brain regional activation changes in rats confronting a snake. Behav. Brain Res. 381, (2020).

86. Cho, J. H., Rendall, S. D. \& Gray, J. M. Brain-wide maps of Fos expression during fear learning and recall. Learn. Mem. 24, 169-181 (2017).

87. Vianna, D. M. L., Borelli, K. G., Ferreira-Netto, C., Macedo, C. E. \& Brandão, M. L. Foslike immunoreactive neurons following electrical stimulation of the dorsal periaqueductal gray at freezing and escape thresholds. Brain Res. Bull. 62, 179-189 (2003).

88. Mongeau, R., Miller, G. A., Chiang, E. \& Anderson, D. J. Neural correlates of competing fear behaviors evoked by an innately aversive stimulus. J. Neurosci. 23, 3855-3868 (2003).

89. Babcock, D. S., Patriquin, H., LaFortune, M. \& Dauzat, M. Power Doppler sonography: basic principles and clinical applications in children. Pediatr. Radiol. 26, 109-115 (1996).

90. Jun, J. J. et al. Fully integrated silicon probes for high-density recording of neural activity. Nature 551, 232-236 (2017).

91. Brainard, D. H. The Psychophysics Toolbox. Spat. Vis. 10, 433-436 (1997).

92. Pelli, D. G. The VideoToolbox software for visual psychophysics: Transforming numbers 
93. Mathis, A. et al. DeepLabCut: markerless pose estimation of user-defined body parts with deep learning. Nat. Neurosci. 21, 1281-1289 (2018).

94. Lopes, G. et al. Bonsai: an event-based framework for processing and controlling data 846 streams . Frontiers in Neuroinformatics 9, 7 (2015).

95. Friston, K. J. et al. Analysis of fMRI time-series revisited. Neuroimage 2, 45-53 (1995).

96. Benjamini, Y. \& Hochberg, Y. Controlling the False Discovery Rate: A Practical and

97. Szekely, G. J. \& Rizzo, M. L. Hierarchical clustering via joint between-within distances:

98. Rousseeuw, P. J. Silhouettes: A graphical aid to the interpretation and validation of

99. Davies, D. L. \& Bouldin, D. W. A Cluster Separation Measure. IEEE Trans. Pattern Anal.

100. Shamash, P., Carandini, M., Harris, K. \& Steinmetz, N. A tool for analyzing electrode

101. Yger, P. et al. A spike sorting toolbox for up to thousands of electrodes validated with 859

102. Segev, R., Goodhouse, J., Puchalla, J. \& Berry, M. J. Recording spikes from a large fraction of the ganglion cells in a retinal patch. Nat. Neurosci. 7, 1154-1161 (2004).

103. Baden, T. et al. The functional diversity of retinal ganglion cells in the mouse. Nature

104. Calinksi, T. \& Harabasz, J. A dendritic method for cluster analysis. Comm. Stat. 3, 1-27 (1974).

866 


\section{Methods}

868 Animals

869 All experimental procedures were approved by the Ethical Committee for Animal

870 Experimentation (ECD) of the KU Leuven and followed the European Communities Guidelines 871 on the Care and Use of Laboratory Animals (004-2014/EEC, 240-2013/EEC, 252872 2015/EEC). Male and female adult (2-4 months old) transgenic mice were used in our 873 experiments including, Ntsr1-GN209Cre, Ai9, Thy1-STOP-YFP, Ai32 x Ntsr1-GN209Cre, Ai32 $874 \times$ PvalbCre and Ai32 x Gad2Cre. Ntsr1-GN209Cre mice (Genset: 030780-UCD) express Cre 875 recombinase in Ntsr1-GN209 expressing neurons. Ai9 (JAX: 007909) and Thy1-STOP876 YFP (JAX: 005630) are reporter lines that express tdTomato and YFP fluorescent proteins 877 respectively, when in presence of the Cre recombinase. Ai32 (JAX: 012569) is a reporter line 878 that expresses Channelrhodopsin2 in presence of Cre recombinase. PvalbCre mice express 879 Cre recombinase in parvalbumin-expressing neurons. Gad2Cre mice express Cre 880 recombinase in Gad2-expressing neurons. Mice were kept on a 12:12 h light:dark cycle and 881 sterilized food pellets and water were provided ad libitum. Experiments were performed during 882 the light phase.

\section{General surgical procedures}

884 Anesthesia was induced at the beginning with an intraperitoneal injection of Ketamine $885(100 \mathrm{mg} / \mathrm{kg})$ and Medetomidine $(1 \mathrm{mg} / \mathrm{Kg})$. Before starting any surgical procedure, the paw of 886 the animal was pinched to check for the absence of pedal reflex. After deep anesthesia was 887 achieved, mice were placed in a stereotaxic workstation (Narishige, SR-5N), on 888 a homeothermic blanket to keep a stable body temperature. Eye ointment was applied to 889 protect the eyes from drying and from light (Dura tear, NOVARTIS, 288/28062-7) and 890 Lidocaine $(0.5 \%, 0.007 \mathrm{mg} / \mathrm{g}$ body weight) was injected under the skin above the skull. The 891 surgical areas were shaved and the skin was disinfected using iso-betadine. Then, the skin 892 was cut following the midline and retracted to the sides to expose the skull. Anterior-posterior 893 coordinates are measured from Bregma.

\section{Viral injections}

895 Once the skull was exposed, a hole was performed at the right coordinates by gently rotating 896 a needle against the skull. We used micropipettes (Wiretrol II capillary micropipettes, 897 Drumond Scientific, 5-000-2005) with an open tip of around $30 \mu \mathrm{m}$, prepared with a Laser898 Based Micropipette Puller (Sutter Instrument, P-2000), and an oil-based hydraulic 899 micromanipulator MO-10 (Narishige) for all stereotactic injections. To trace back the injection 900 sites, we coated the glass pipette tip with DiD (Thermo, D7757).

901 For optogenetic experiments, we targeted Camkll-expressing neurons of the retinorecipient 902 layers of the colliculus by injecting wild type mice with AAV2-Camkll-hChR2(E123T/T159C)903 p2A-EYFP-WPRE (UNC vector core, AV5456B). We used Ai9 and Thy1-STOP-YFP mice as 904 wild type mice. We injected 200-300 nl of AAV in $100 \mathrm{nl}$ doses with a waiting time of 5-10 min 905 after each injection. Coordinates for the superficial colliculus were AP: -3.6 to 3.8, ML: -0.2 to $906-0.3$, DV: -1.1 to -1.4 . To express ChR2 specifically in Ntsr1-GN209 expressing neurons of the 

WPRE (UNC vector core, AV5468C) into the superficial colliculus of Ntsr1-GN209Cre mice.

909 For chemogenetic experiments, we injected $300 \mathrm{nl}$ of AAV2-hSyn-hM4D(Gi)-mCherry 910 (Addgene, 50475), or AAV2-hSyn-EYFP (UNC vector core, AV4376E) as control, into the 911 PPnT. PPnT coordinates: AP -3 to $-3.4, \mathrm{ML}-1.8$ to 2, DV: -3.5 to -3.3 .

912 Following injection, the skin was glued with Vetbond tissue adhesive $(3 \mathrm{M}, 1469)$ to close the 913 wound. Next, mice were injected with painkillers (Buprenorphine $0.2 \mathrm{mg} / \mathrm{kg} \mathrm{I.P.)} \mathrm{and} \mathrm{antibiotics}$ 914 (Cefazolin $15 \mathrm{mg} / \mathrm{kg}$ I.P.) and were allowed to recover on top of a heating pad. After recovery 915 from anesthesia, animals were provided with soft food and water containing antibiotics 916 (emdotrim, ecuphar, BE-V23552) and were monitored for 3 days and administrated 917 Buprenorphine and Cefazolin depending on the condition of the animal. Any following surgery 918 was performed 21 days after injection to allow for proper gene expression.

\section{Cranial Windows and optic-fiber cannula Implantations}

920 opto-fUSi

921 Once the mouse was anesthetized and the skull was exposed, the lateral and posterior muscles were retracted. Vetbond was applied to open skin and exposed muscle, and a titanium head plate was attached to the skull using dental cement (Superbond C\&B, Prestigedental). Then a cranial window extending over almost the whole extent of the left hemisphere and part of the right hemisphere (AP: +2 to $-6.5 \mathrm{~mm}, \mathrm{ML} 1.5$ to -4.5 ) was made with a drill. Then, an optic-fiber cannula (Doric Lenses, MF1.25, 200/245-0.37, FLT) was implanted. The entry point of the fiber into the brain was AP: -3.6 to $-3.8, M L+1.5$ at a $56^{\circ}$ angle. The fiber was slowly inserted $1.8 \mathrm{~mm}$ into the brain so that the tip would be placed at ML: 0 and DV: -1.1 to -1.2. Next, a ring of dental cement was formed around the craniotomy and the optic-fiber to stabilize the whole preparation. Finally, the cranial window was covered with silicone elastomer for protection and the mouse was allowed to recover on a heating pad. Mice were treated with painkillers (Buprenorphine $0.2 \mathrm{mg} / \mathrm{kg}$ I.P.), antibiotics (Cefazolin $15 \mathrm{mg} / \mathrm{kg}$ I.P.) and antiinflammatory (Dexamethasone $0.1 \mathrm{mg} / \mathrm{kg}$ ) drugs for 5 days.

In vivo electrophysiology

935

Once the mouse was anesthetized and the skull was exposed, Vetbond was applied to open skin and exposed muscle, and a titanium head plate was attached to the skull using dental cement (Superbond C\&B, Prestige-dental). Then, cranial windows $\left(\sim 0.5\right.$ to $\left.1 \mathrm{~mm}^{2}\right)$ were performed over the coordinates of the target regions. The following coordinates were used as the center of craniotomies: SC: AP: -3.7, ML: -0.5; PPnT: AP: -3.3, ML: -1.9; lateral posterior nucleus of the thalamus: AP: -2.1 , ML: -1.8 ; tail of caudate putamen: AP: -1.4 , ML: -3.1 . An additional hole was made for the implantation of an optic-fiber cannula. The entry point of the fiber into the brain was AP: -3.6 to $-3.8, \mathrm{ML}:+1.5$ at a $56^{\circ}$ angle. The fiber was slowly inserted $1.8 \mathrm{~mm}$ into the brain so that the tip would be placed at ML: 0 and DV: -1.1 to -1.2 .

\section{Opto-open field test}

945 All behavioral experiments were performed in a custom made square wooden box (W: $50 \mathrm{~cm}$ $946 \times$ L: $50 \mathrm{~cm} \times \mathrm{H}: 36 \mathrm{~cm}$ ). Dim ambient light ( 50 lux) was provided by a lamp (Paulmann Licht 
$947 \mathrm{GmbH}$, PDG09/14) positioned above the arena and oriented away from it, towards a wall.

948 Behavior was recorded at $30 \mathrm{fps}$ using a camera (Point Grey Research, FMVU-03MTM-CS)

949 positioned $53 \mathrm{~cm}$ above the center of the arena. For optogenetic activation we used a $473 \mathrm{~nm}$

950 DPSS laser system (Laserglow Technologies, R471003GX) connected to a patchcord with a

951 rotatory joint (Thorlabs, RJPFL2). Optogenetic stimulation was controlled with custom software

952 written in MATLAB. Before every experiment, the output of the laser was measured at $20 \mathrm{~Hz}$

953 or $50 \mathrm{~Hz}$ (2 ms pulse width) and set at 0.3-0.4 mW $\left(9.5-12.5 \mathrm{~mW} / \mathrm{mm}^{2}\right)$. For any given mouse

954 line, a high-frequency stimulus was chosen based on preliminary behavioral data. In those

955 mouse lines where $20 \mathrm{~Hz}$ stimulation did not evoke any visible response, the following

956 experiments were done at $50 \mathrm{~Hz}$. In the data shown here, CAMKII and NTSR mice were

957 stimulated at $20 \mathrm{~Hz}$ whereas PV and GAD2 were stimulated at $50 \mathrm{~Hz}$.

9585 days after implantation of the optic fiber, mice were habituated to the handler, patchcord and 959 experimental room for at least 3 days. The day of the test, mice were placed in the center of 960 the arena and were allowed to freely explore for $2 \mathrm{~min}$. After the acclimatization time, when 961 the mice moved away $(\sim 10 \mathrm{~cm})$ from the perimeter of the box, towards the center, light 962 stimulation was manually triggered. At any given test, mice where stimulated at high ( $20 \mathrm{~Hz}$ or $96350 \mathrm{~Hz}, 20$ or 50 pulses) and low ( $5 \mathrm{~Hz}, 20$ pulses) frequencies in a pseudo-random manner. 964 Time between stimuli was set to be of at least 30 seconds. A typical experiment lasted 20-40 965 min.

966 In tests that combined optogenetics with DREADDs, the experiments where performed as 967 explained above, except that mice were injected with either CNO (2 mg/kg) or saline $30 \mathrm{~min}$ 968 prior to the test and where only stimulated at $20 \mathrm{~Hz}$.

969 Repeated tests where always separated by at least $48 \mathrm{~h}$.

970 Protocol of Functional Ultrasound Imaging

9715 days after surgery, mice where habituated to the handler, experimental room and to head-

972 fixation on a platform for 7 days. Then, the awake mouse was head-fixed on the platform and 973 the body movement was partially restrained by a foam shelter. The silicone cap was removed 974 and the cranial window was covered with a 2-3 \% agarose layer to reduce brain movement. A $975473 \mathrm{~nm}$ DPSS laser system was then connected to the optic fiber cannula using a ferrule patch 976 cable (Thorlabs, M83L01). Before every experiment, the output of the laser was measured at $97720 \mathrm{~Hz}$ or $50 \mathrm{~Hz}$ and set at 0.3-0.4 mW (9.5-12.5 mW/mm2). Next, acoustic gel ( 1 mL, Unigel, 978 Asept) was applied on the agarose for ultrasound coupling and the ultrasound probe (L22-14v, 979 Verasonics) was lowered down to a distance of $\sim 3 \mathrm{~mm}$ from the brain. The probe was moved 980 along the lateral axis by a linear microprecision motor (Zaber, X-LRM-DE). At the beginning of 981 each session, a reference anatomical scan was acquired for registration (53 sagittal planes 982 from lateral $+1.5 \mathrm{~mm}$ to $-5 \mathrm{~mm}, 125 \mu \mathrm{m}$ steps). Following, we acquired the functional scan (23 983 sagittal planes, from lateral $+1.5 \mathrm{~mm}$ to $-4.5 \mathrm{~mm}, 250 \mu \mathrm{m}$ steps). Two optogenetic stimuli were 984 applied at each plane (high and low frequencies) before moving to the next one. For each 985 stimulus, functional images were acquired for $20 \mathrm{~s}(10 \mathrm{~Hz})$, and the stimulus was applied after 986 a $10 \mathrm{~s}$ baseline. The functional imaging and optogenetic stimulation were controlled and 
synchronized using custom software written in MATLAB. Optogenetic stimuli consisted of a high ( $20 \mathrm{~Hz}, 20$ pulses, $2 \mathrm{~ms}$ pulse width or $50 \mathrm{~Hz}, 50$ pulses, 2 ms pulse width) and a low (5 $\mathrm{Hz}, 20$ pulses, $2 \mathrm{~ms}$ pulse width, $\sim 2 \mathrm{~mW} / \mathrm{mm}^{2}$ ) frequency stimulus. The acquisition of the 23 sagittal planes was acquired sequentially starting at lateral -4.5 , and the whole craniotomy was imaged 7-12 times per session. Total acquisition time was $\sim 3.5 \mathrm{~h}$.

\section{Generation of a Functional Ultrasound Image}

993 This procedure was adapted from the sequence for fast, whole-brain functional ultrasound 994 imaging described in Macé et al., 2018. An ultrasound probe containing a linear array of 128 ultrasound emitters/receivers, emitted plane waves (15 MHz, 2 cycles) in five different angles

$996\left(-6^{\circ},-3^{\circ}, 0^{\circ}, 3^{\circ}, 6^{\circ}\right)$. The echoes from each plane wave was acquired with the receivers and 997 adjusted with a time-gain compensation to account for the attenuation of ultrasound signals 998 with depth (exponential amplification of $1 \mathrm{~dB} / \mathrm{mm}$ ). This process generated a single emit999 receive image ('B-mode image') for each angle and was applied three times for averaging. The 15 individual B-mode images were then combined $(\sim 2 \mathrm{~ms}, 500 \mathrm{~Hz})$, resulting in a higher quality image ('compound B-mode image').

50 compound B-mode images were acquired every $100 \mathrm{~ms}(10 \mathrm{~Hz})$ to generate a functional ultrasound image. Blood cells flowing inside the vessels scatter back and shift the frequency of the emitted waves (Doppler effect). Such shifts were measured and extracted in real-time, using singular-value-decomposition-based spatiotemporal filtering, and high-pass temporal filtering (cut-off frequency: $20 \mathrm{~Hz}$ ). From the filtered data, we calculated the mean intensity of the Doppler signal (Power Doppler) in each voxel. Power Doppler integrates all the Doppler signals in a voxel to obtain an intensity value that is proportional to the amount of blood cells moving in that voxel at a given time. Unlike Color Doppler, it lacks information about velocity or direction of the blood flow but reliably reports hemodynamic changes in blood volume ${ }^{27,40,89}$. The intensity value of a voxel at a given time was calculated as: $I(x, y)=A(x, y, t)^{2}$ where $l$ is Power Doppler Intensity, $x, y$ are the coordinates of a given voxel in a given plane, $A$ is the amplitude of the compound B-mode images after filtering, and $t$ was time. The resulting functional ultrasound image was $143 \times 128$ voxels in which each voxel had a size of $52.5 \mu \mathrm{m} \mathrm{x}$ $100 \mu \mathrm{m} \times 300 \mu \mathrm{m}^{28}$.

\section{Electrophysiological recordings}

1017 12 NTSR1-GN209-Cre $\times$ Chr2 (Ai32) mice of either sex at the age of 2.5-3 months were used 1018 to record optogenetic and light driven responses in the superior colliculus and PAG (7 1019 recordings), pulvinar (9 recordings), caudateputamen (9 recordings) and posterior 1020 paralaminar nuclei of the thalamus (3 recordings).

1021 Two days after performing cranial windows, animals were habituated to the recording set up 1022 for 3-4 days. The day of the recording, head-posted animals were fixed on a treadmill or 1023 floating ball in front of a screen. Then, a Neuropixels probe phase 3A (Imec, Belgium) ${ }^{90} \mathrm{coated}$ 1024 with a fluorescent dye (DiD, Thermofisher) was inserted into the brain with the tip reaching 1025 down to 1-1.5 mm below the target area. Once the right depth was reached, it was left to rest 1026 for 20-30 min, before starting the recording. Artificial cerebrospinal fluid (150 mM NaCl, $5 \mathrm{mM}$ 

to $\mathrm{pH} 7.4$ with $\mathrm{NaOH}$ ) was used to cover the exposed brain and skull.

1029 Neuropixel probes contain 960 recording sites on a single shaft distributed in two rows of 480 1030 electrodes along $9600 \mu \mathrm{m}$ ( $16 \mu \mathrm{m}$ lateral spacing, $20 \mu \mathrm{m}$ vertical spacing), of which $384 \mathrm{can}$ 1031 be recorded simultaneously. In all our experiments, we recorded from the 384 electrodes closest to the tip, spanning $3840 \mu \mathrm{m}$. Signals were recorded at $30 \mathrm{KHz}$ using the Neuropixels headstage (Imec), base station (Imec) and a Kintex-7 KC705 FPGA (Xilinx). High-frequencies $(>300 \mathrm{~Hz})$ and low-frequencies $(<300 \mathrm{~Hz})$ were acquired separately. To select the recording electrodes, adjust gain corrections, observe online recordings, and save data, we used SpikeGLX software. Timings of visual and optogenetic stimulation were recorded simultaneously using digital ports of the base station.

While recording from any given location, first, the superior colliculus of these mice received 30 repetitions of blue light trains $(20 \mathrm{~Hz}, 20$ pulses, 1 ms pulse width, $0.2 \mathrm{~mW})$ spaced by 20 seconds intervals. Then, visual stimuli were presented.

1041

\section{Visual stimuli}

1042

Visual stimuli were presented on a 32-inch LCD monitor (Samsung S32E590C, 1920x1080 pixel resolution, $60 \mathrm{~Hz}$ refresh rate, average luminance of $\left.2.6 \mathrm{~cd} / \mathrm{m}^{2}\right)$ positioned perpendicular to the mouse head, at $35 \mathrm{~cm}$ from the right eye, so that the screen was covering $90^{\circ}$ of azimuth and $70^{\circ}$ of altitude of the right visual field. Visual stimuli were presented on a gray background (50\% luminance), controlled by Octave (GNU Octave) and Psychtoolbox ${ }^{91,92}$. The visual stimuli consisted of a small black disc that linearly expanded from $2^{\circ}$ to $50^{\circ}$ of diameter within $300 \mathrm{~ms}$ at the center of the screen and a disk of $50^{\circ}$ diameter dimming from background to black within $300 \mathrm{~ms}$. The stimuli were repeated 10 times.

\section{Immunohistochemistry}

1051 Animals were perfused and post-fixed overnight using 4\% paraformaldehyde (HistoFix, Roche). Vibratome sections (100-200 $\mu \mathrm{m})$ were collected in 1x PBS and were incubated in blocking buffer (1x PBS, 0.3\% Triton X-100,10\% Donkey serum) at room temperature for 1 hour. Then slices were incubated with primary antibodies in blocking buffer overnight at $4^{\circ} \mathrm{C}$. The next day, slices were washed 3 times for 10 min each in 1x PBS with $0.3 \%$ TritonX-100 and incubated in secondary antibody solution diluted in blocking buffer overnight at $4^{\circ} \mathrm{C}$. We used rabbit anti-GFP (Thermo Fisher, A-11122, 1:500) as a primary antibody to label Chr2positive cells and anti-mCherry (Novus, NBP2-25158, 1:500) to label hM4D-postive cells. Alexa488 donkey anti-rabbit (Thermo Fisher, A21206, 1:500-1000) and Cy3 donkey antichicken (ImmunoJackson, 703-166-155, 1:1000) were used as secondary antibodies. Nuclei were stained with DAPI (Roche, 10236276001, 1:500) together with the secondary antibody solution. Sections were then again washed 3 times for $10 \mathrm{~min}$ in $1 \times$ PBS with $0.3 \%$ TritonX100 and 1 time in 1x PBS, covered with mounting medium (Dako, C0563) and a glass coverslip. Confocal microscopy was performed on a Zeiss LSM 710 microscope. Images of areas with Chr2- and hMD4-expressing cells, the fiber location and the Neuropixels track labelled with DiD were obtained using a 10x (plan-APOCHROMAT 0.45 NA, Zeiss) objective. 
1067 Analysis of behavioral data

1068 Animal tracking was performed using DeeLabCut software ${ }^{93}$. Stimulus onsets and offsets were 1069 extracted with custom-made Bonsai workflow ${ }^{94}$. Tracking data were sorted into peri-stimulus 1070 trials using custom made Python scripts. Trials where stimulation happened in the periphery 1071 of the setup were not included in the analysis, unless explicitly stated. Behavioral parameters were calculated by pooling all trials per mouse and calculating the average, followed by average over mice. Speed was extracted based on positional data of the base of the tail obtained from DeepLabCut. Frames with probability lower than 0.9 were filtered out and linearly interpolated. Position data were smoothed with a median filter of window size $=5$. The preferred body angle (Figure 1G) was obtained by first aligning the trajectories to the same initial position and by rotating them to Cartesian $X$ axis by an angel of their body position at the stimulus onset frame. The body position angle is the angle between the line connecting the tail base with the nose and the Cartesian X axis. Next, the preferred angle was calculated between then line of stimulus onset nose position and stimulus offset nose position. Latency to the corner was analyzed as time needed to reach a corner (corner is defined as a square of $10 \times 10 \mathrm{~cm}$ ) after the stimulation onset.

\section{Analysis of fUSi data}

1084 Registration

1085 At the beginning of each session, we acquired a reference anatomical map. These anatomical maps were then registered to the Allen Mouse Brain Common Coordinate Framework version 3 (CCFv3) (Allen Brain API; brain-map.org/api/index.html) (Figure 2D). Registration was done semi-automatically based on anatomical landmarks that could be recognized on both the anatomical map and CCFv3 (external outline of the brain, dorsal hippocampus, 3rd and 4th ventricles, cerebellar outline, middle cerebral sinus, colliculus, and corpus callosum). These landmarks were used to readjust the 3D volume of the reference map to the CCFv3 by applying scaling in either of the $x, y, z$ axis and rotations and translations in the coronal, sagittal or axial planes when necessary. Then, we calculated the rotations and translations of the coronal, sagittal and axial planes to create a 3D transformation matrix (from anatomical map to CCFv3).

\section{Segmentation}

1096 fUSi from each session were automatically registered to the CCFv3 using the 3D 1097 transformation matrices obtained in the previous steps. Assignment of voxels to brain areas 1098 was based on the CCFv3 segmentation. For our analysis, we excluded fiber tracts, ventricles, 1099 unsegmented parts of main brain structures (CTX, CTXsp, TH, HY, MB, HB, CB), merged 1100 layers of brain areas, and excluded or merged neighboring areas with volumes $<300 \mu \mathrm{m}^{3}$. Our 1101 final version of the atlas was comprised of 264 brain areas in one hemisphere of the brain (see 1102 Table S2).

1103 Response Time Traces

1104 The relative hemodynamic changes $(\Delta \mathrm{I} / \mathrm{I})$ were calculated for each voxel, where I was the 1105 baseline (mean of $10 \mathrm{~s}$ before stimulus onset) and $\Delta \mathrm{l}$ was the subtraction of the baseline to the 
signal at each time point. The traces of the individual areas were obtained for every individual trial by summing all the voxels assigned to each area (Figure $2 \mathrm{E}$ ).

\section{Data filtering and normalization.}

In order to analyze the response traces of each segmented area, first, we created a dataset with the temporal signals, $\mathrm{Ti} i=1 . . \mathrm{Ntime}$, of each region, trial and session of each animal; $\mathrm{T}_{\text {Animal,repetition }}$ (region,time). In this dataset, all the trials of the different sessions were added. Therefore, the repetitions were Nsessions*Ntrials. The intensity signal obtained from the Power doppler, is susceptible to brain tissue motions caused by the awake animal's movements. However, motion artifacts can typically be distinguished from hemodynamic changes based on the shape (noise/real; quick spiky/slow curved), and amplitude (noise/real $\Delta \mathrm{l} / \mathrm{l} ;>100 \% / 1-15 \%)$ of the signal. In this study, in order to remove trials affected by motion artifacts we computed the mean temporal signal of each region $(\mathrm{Tm})$ and standard deviation (SD), and then eliminated outlier values where Ti-Tm were 2.5 times higher than the SD. The eliminated values were replaced by the previous non-eliminated value. To eliminate the global variations in the brain (baseline perturbations) we selected the $20 \%$ of the regions with lowest response during a 2 seconds time window after the stimulus onset, then averaged these regions to create a baseline signal. This baseline signal was then subtracted from all segmented areas. The resulting normalized temporal traces where used for statistical analysis (Figure 2G).

\section{Active Brain Regions}

To determine if a brain region was activated by a stimulus, first, we used the normalized temporal traces of every trial to calculate a T-score for each animal, using a general linear model, as commonly used in $\mathrm{fMRI}{ }^{95}$. To take into account the different temporal dynamics present in the responses, the GLM was applied using 1 and 2 seconds stepped ( 0.5 seconds steps) time windows, starting at the stimulus onset until 7.5 seconds after stimulus. Next, a one-sample two-tailed $t$ test was performed on the $n$ T-scores obtained for the $n$ animals. The region was considered active if the resulting $P$-value, adjusted for a false discovery rate (Benjamini and Hochberg FDR procedure), was $<0.05$ (Figure S4D) ${ }^{96}$. For display (such as in Figure $2 \mathrm{~J}$ ), we quantified the median response time courses across animals, we standardized the responses with regard to the values before stimulus onset (z-score) and we corrected for the relative differences between the stimulation levels of each animal. To do this we calculated a correction factor from normalizing the peak response $(A=$ average of the signal 0.5 s around the maximal value within a time window after stimulus onset), across all brain areas where: A_norm(region) $=A($ region $) /$ sqrt(sum $\left(A(\text { region })^{2}\right)$, then divided the correction factor across each time point. The Average of all the corrected response traces, for each mouse line, are shown in Figure $2 \mathrm{~J}$ and $\mathrm{S} 2 \mathrm{G}$ and are used in the subsequent figures.

\section{Pixel-to-pixel Activity Maps}

To visualize pixel-to-pixel activity maps, for each voxel, we quantified the median of the response time courses across trials. We then standardized the responses computing the $z-$ score, using the 10 seconds before stimulus onset and a 2 seconds time window after stimulus 
onset. Next, we averaged activity maps of animals belonging to the same mouse line applied

1147 a median filtering of $4 \times 4$ pixels on the resulting z-score map. The filtered $z$-score maps were

1148 used for visual inspection of the brain activity and for data visualization (Figure $5 \mathrm{H}$ ).

1149 Clustering of fUSi time courses

1150 Time course of all active brain regions in the four neuronal groups were clustered using

1151 hierarchical classification and the e-linkage algorithm, an extensions of Ward's minimum

1152 variance method ${ }^{97}$. The optimal number of clusters was determined by visual inspection of the 1153 mean silhouette value ${ }^{98}$ and Davies-Bouldin Index ${ }^{99}$. Clustering was only preformed on the 11541056 active areas for the 1s optogenetic stimulus.

\section{Analysis of Neuropixels recordings}

\section{Raw spiking activity}

1157 To extract spikes from raw Neuropixels data, the average voltage on each electrode in the

$11580.5 \mathrm{~s}$ before onset of the optogenetic stimulation was subtracted from the signal during 1159 stimulation. Spikes were identified using the 'findpeaks' function in MATLAB with a threshold 1160 of 4 standard deviations of the signal before the stimulation. Spikes during the $1 \mathrm{~ms}$ of each 1161 light pulse were excluded as they could cause artifacts, especially on the electrodes in the 1162 superior colliculus.

1163 Activity maps

1164 Confocal images of brain slices containing the probe tracks were aligned with the Allen Brain 1165 Atlas using the allen CCF tool ${ }^{100}$. This allowed us to identify the same slice of the fUSi data 1166 set. To compare the activity on the probe with the fUSi signal, we averaged the z-scored signal 1167 of $x-2$ to $x+2$ pixels for each $x$ location of the probe. The raw spiking data on the whole probe 1168 was resampled to match the resolution of the fUSi data. We normalized each data set 1169 separately to its maximal value, resulting in the plot shown in Figure 6G and analyzed in Figure $11706 \mathrm{~F}$. Correlation coefficients were calculated using the 'corrcoef' function in MATLAB.

\section{Spike sorting}

1172 The high-pass filtered in-vivo data was automatically sorted into individual units using SpyKING 1173 CIRCUS ${ }^{101}$ with the following parameters: cc_merge $=0.95$ (merging if cross-correlation 1174 similarity $>0.95$ ), spike_thresh $=6.5$ (threshold for spike detection), cut_off $=500$ (cut-off 1175 frequency for the butterworth filter in $\mathrm{Hz}$ ). Automated clustering was followed by manual 1176 inspection, merging of units if necessary and discarding of noise and multi-units using phy2 1177 (https://github.com/cortex-lab/phy). Units were evaluated based on the average waveform 1178 shape and auto-correlogram. Only cells with $<1 \%$ of inter-spike intervals of $\leq 1$ ms were 1179 considered and cross correlograms with nearby neurons were inspected to find spikes from 1180 the same neurons ${ }^{102}$.

1181 Detection of responding units

1182 Peri-stimulus histograms (PSTH) were calculated using a bin size of $20 \mathrm{~ms}$. For detection of 1183 responding cells and for plotting, the mean spikes/s during 0.3 (looming, dimming) or 0.5 1184 seconds (optogenetic stimulus) before stimulus onset were subtracted from the cell's activity. 
1185 We calculated a quality index to capture the reliability of a cell's response to the 10-30 stimulus 1186 repetitions. The quality index was defined as $Q I=\frac{\operatorname{Var}\left[\bar{C}_{r}\right]_{t}}{\overline{\operatorname{Var}\left[C_{t}\right]_{r}}}$ with $\mathrm{C}$ being the TxR response 1187 matrix, $\mathrm{t}=$ time dimension and $\mathrm{r}=$ repetition dimension ${ }^{103}$. Cells were labelled as 'responding' 1188 if the maximal z-score during the stimulus exceeded 3 and if the quality index was at least 11890.15.

1190 Transiency measurements

1191 For response transiency when stimulated with 20 light pulses (optogenetics), the mean 1192 response during the $\mathbf{4 0} \mathrm{ms}$ after onset of each pulse was normalized to the absolute maximum 1193 of these 20 responses (positive or negative). The transiency of the response was defined as 1194 the area under the curve (AUC), i.e. the sum of these 20 values divided by 20. An AUC of 1 1195 means that the cell responded equally well to all 20 pulses, an AUC of -1 means that the cell's 1196 activity was equally suppressed by each pulse.

\section{Clustering of optogenetically induced responses}

1198 The normalized responses to the 20 light pulses were clustered using k-means (MATLAB) with 1199 squared Euclidean distance measurement and 1000 replicates. Based on the Calinski1200 Harabasz ${ }^{104}$ and Davies-Bouldin indices ${ }^{99}, 7$ clusters were chosen where one cluster only 1201 contained very few cells with stronger inhibition. These cells were merged with another cluster 1202 consisting of inhibited cells, resulting in 6 total clusters. 